\title{
Measuring Anti-Factor Xa Activity to Monitor Low-Molecular-Weight Heparin in Obesity: A Critical Review
}

\author{
Gregory Egan and Mary H H Ensom
}

\begin{abstract}
Background: The choice of whether to monitor anti-factor Xa (anti-Xa) activity in patients who are obese and who are receiving low-molecularweight heparin (LMWH) therapy is controversial. To the authors' knowledge, no systematic review of monitoring of anti-Xa activity in such patients has been published to date.
\end{abstract}

Objective: To systematically ascertain the utility of monitoring anti-Xa concentrations for LMWH therapy in obese patients.

Data Sources: MEDLINE (1946 to September 2014), the Cochrane Database of Systematic Reviews, Embase (1974 to September 2014), PubMed (1947 to September 2014), International Pharmaceutical Abstracts (1970 to September 2014), and Scopus were searched using the terms obesity, morbid obesity, thrombosis, venous thrombosis, embolism, venous thromboembolism, pulmonary embolism, low-molecular weight heparin, enoxaparin, dalteparin, tinzaparin, anti-factor Xa, anti-factor Xa monitoring, anti-factor Xa activity, and anti-factor Xa assay. The reference lists of retrieved articles were also reviewed.

Study Selection and Data Extraction: English-language studies describing obese patients treated with LMWH or reporting anti-Xa activity were reviewed using a 9-step decision-making algorithm to determine whether monitoring of LMWH therapy by means of anti-Xa activity in obesity is warranted. Studies published in abstract form were excluded.

Data Synthesis: The analysis showed that anti-Xa concentrations are not strongly associated with thrombosis or hemorrhage. In clinical studies of LMWH for thromboprophylaxis in bariatric surgery, orthopedic surgery, general surgery, and medical patients, and for treatment of venous thromboembolism and acute coronary syndrome, anti-Xa activity can be predicted from dose of LMWH and total body weight; no difference in clinical outcome was found between obese and non-obese participants.

Conclusions: Routinely determining anti-Xa concentrations in obese patients to monitor the clinical effectiveness of LMWH is not warranted on the basis of the current evidence. Circumstances where measurement of anti-Xa concentration may help in clinical decision-making in either obese or non-obese patients would be cases where elimination of LMWH is impaired or there is an unexpected clinical response, as well as to confirm compliance with therapy or to identify deviation from predicted pharmacokinetics.

Keywords: low-molecular-weight heparin, anti-factor Xa, anti-Xa, therapeutic drug monitoring

\section{RÉSUMÉ}

Contexte : Choisir d'effectuer ou non une surveillance de l'activité de l'anti-facteur Xa (anti-Xa) chez le patient obèse qui reçoit un traitement par héparine de bas poids moléculaire (HBPM) est controversé. À la connaissance des auteurs, aucune analyse systématique de la surveillance de l'activité anti-Xa chez ce type de patient n'a été publiée à ce jour.

Objectif : Établir systématiquement l'utilité de la surveillance des concentrations d'anti-Xa pour le traitement par HBPM chez le patient obèse.

Sources des données : Les bases de données MEDLINE (de 1946 à septembre 2014), Cochrane Database of Systematic Reviews, Embase (de 1974 à septembre 2014), PubMed (de 1947 à septembre 2014), International Pharmaceutical Abstracts (de 1970 à septembre 2014) et Scopus ont été interrogées à l'aide des termes obésité, obésité morbide, thrombose, thrombose veineuse, embolie, événement thromboembolique veineux, embolie pulmonaire, héparine de bas poids moléculaire, énoxaparine, daltéparine, tinzaparine, anti-facteur Xa, surveillance de l'anti-facteur Xa, activité anti-facteur Xa et analyse de l'activité anti-facteur Xa. Un examen des bibliographies des articles extraits a aussi été réalisé.

Sélection des études et extraction des données : Les études en anglais présentant des patients obèses traités par HBPM ou signalant l'activité anti-Xa ont été examinées à l'aide d'un algorithme de prise de décision à neuf étapes dans le but de déterminer s'il est justifié de réaliser une surveillance des HBPM en mesurant l'activité anti-Xa dans les cas d'obésité. Les études publiées sous forme de résumé étaient exclues.

Synthèse des données : Les concentrations d'anti-Xa ne sont pas fortement associées aux thromboses ou aux hémorragies. Dans les études cliniques sur la thromboprophylaxie en chirurgie bariatrique, en chirurgie orthopédique, en chirurgie générale et chez le patient médical, et sur le traitement de la thromboembolie veineuse et du syndrome coronarien aigu, l'activité anti-Xa peut être prédite à l'aide de la dose de HBPM et du poids total du patient. Aucune différence dans les résultats cliniques entre les sujets obèses et non obèses n'a été trouvée.

Conclusions : À la lumière des données probantes actuelles, il n'est pas justifié d'effectuer une analyse systématique des concentrations d'anti-Xa chez le patient obèse afin de surveiller l'efficacité clinique des HBPM. Il reste tout de même des situations pour lesquelles l'analyse des concentrations d'anti-Xa chez le patient obèse ou non obèse pourrait aider à prendre une décision clinique : présence d'une élimination déficiente des HBPM, survenue d'une réaction clinique inattendue, confirmation de l'observance du traitement ou explication d'un écart de la pharmacocinétique prévue. 
Can J Hosp Pharm. 2015;68(1):33-47
Mots clés : héparine de bas poids moléculaire, anti-facteur Xa, anti-Xa, suivi thérapeutique pharmacologique

\section{INTRODUCTION}

$\mathrm{H}$ eparin, a sulfated polysaccharide, is widely used as an anticoagulant to treat and prevent thromboembolic disease. ${ }^{1}$ Unfractionated heparin (UFH) preparations, consisting of heparin fragments of various lengths, have a mean molecular weight of 12000 to 15000 daltons (range 3000 to 35000 daltons). ${ }^{1-3}$ Although quite effective for its intended purpose, UFH has been associated with paradoxical thrombosis, and its use entails continuous IV administration with routine monitoring of coagulation via tests for activated partial thromboplastin time (aPTT) or anti-factor Xa (anti-Xa). ${ }^{2-4}$ As an alternative to UFH, low-molecular-weight heparin (LMWH) preparations were first introduced in the early 1980 s. ${ }^{2,5}$ Initial pharmacodynamic studies showed that heparin fragments with a molecular weight less than 6000 daltons do not prolong aPTT but do inactivate factor $\mathrm{Xa} .^{1,2,5-7}$ Early trials in the treatment and prevention of venous thromboembolism (VTE) showed that LMWH produces an antithrombotic effect equivalent to that of UFH..$^{8-12}$ Since then, the use of LMWH has increased dramatically, as the shorter fragment length confers pharmacodynamic and pharmacokinetic advantages. ${ }^{13-15}$

In vivo, heparin fragments exert an anticoagulant effect by forming complexes with antithrombin III and then binding and inactivating coagulation factors IIa and Xa. ${ }^{1,2,4}$ The affinity of the heparin-antithrombin III complex for factor IIa depends on fragment length; more specifically, an 18-polysaccharide chain is necessary for inactivation of factor IIa. ${ }^{1,2,4}$ Longer saccharide chains (>18 units) account for more than 50\% of LMWH fragments, and the anti-Xa activity of these longer chains is greater than their anti-factor II activity. ${ }^{5}$ Heparin also inactivates factors VIIa, IXa, Xia, and XIIa, although the importance of these factors to anticoagulant effect is debated and they are therefore not monitored..$^{4-7}$ Also, LMWH fragments do not have the same propensity to interact with endothelial cells and plasma proteins, and they stimulate release of von Willebrand factor to a lesser extent than does UFH. ${ }^{4-7}$ Clinically, the anticoagulant effect of LMWH is monitored by measuring anti-Xa concentration. ${ }^{3,4-6,16}$

The pharmacokinetic parameters of LMWH are well defined for normal-weight individuals. The volume of distribution $\left(V_{\mathrm{d}}\right)$ ranges from 0.05 to $0.07 \mathrm{~L} / \mathrm{kg}$, depending on the specific fragment, and is approximated by the intravascular volume. ${ }^{17-19}$ The plasma half-life is $4-7 \mathrm{~h}$, LMWH is almost entirely renally cleared, and it undergoes first-order elimination. ${ }^{17-20}$ In obese patients, the difference in body composition may affect the pharmacokinetic parameters of LMWH. ${ }^{21}$ For example, the absorption of LMWH administered subcutaneously may be prolonged in obesity. If $V_{\mathrm{d}}$ is limited to intravascular volume, then dosing by total body weight in obese patients may result in greater elevation of anti-Xa concentration than anticipated and could thereby increase the risk of hemorrhage. ${ }^{8,16,21}$ Renal clearance of LMWH may be increased in obesity because of increased renal blood flow resulting in lower-than-expected anti-Xa concentrations and increasing the risk of thrombosis. ${ }^{8,16,21}$

As a result of the limited pharmacokinetic data available from obese patients, anti-Xa monitoring of LMWH therapy is recommended when total body weight exceeds $150 \mathrm{~kg}$. 3,8,17-19,22-24 This rationale has been challenged by the authors of previous reviews, who suggested that LMWH can be safely dosed by total body weight up to $190 \mathrm{~kg}$. $^{8,17-19,22-24}$ However, to the current authors' knowledge, a systematic review of the monitoring of anti-Xa activity in obese patients has not been published previously. The objective of this review was to systematically ascertain the utility of monitoring anti-Xa concentrations for LMWH therapy in obese patients.

\section{SEARCH STRATEGY}

The systematic search, for English-language publications only, was applied to MEDLINE (1946 to September 2014), the Cochrane Database of Systematic Reviews, EMBASE (1974 to September 2014), PubMed (1947 to September 2014), International Pharmaceutical Abstracts (1970 to September 2014), and Scopus. The search used the following Medical Subject Headings (MeSH terms) related to obesity, VTE, LMWH, and anti-Xa concentration: obesity, morbid obesity, thrombosis, venous thrombosis, embolism, venous thromboembolism, pulmonary embolism, low-molecular weight heparin, enoxaparin, dalteparin, tinzaparin, anti-factor Xa, anti-factor Xa monitoring, anti-factor $\mathrm{Xa}$ activity, and anti-factor Xa assay (see Box 1 for details about how the terms were combined in the searches). Bibliographies of included studies were screened for additional titles that met study selection criteria. 


\section{Box 1. Combinations of Medical Subject Headings (MeSH Terms) Used in the Search Strategy}

Databases were searched using the following terms:

"obesity" or "morbid obesity"

("thrombosis" or "venous thrombosis" or "embolism") and ("thrombosis" or "venous thromboembolism" or "pulmonary embolism")

"low-molecular weight heparin" or "enoxaparin" or "dalteparin" or "tinzaparin"

"anti-factor Xa" or "anti-factor Xa monitoring" or

"anti-factor Xa activity" or "anti-factor Xa assay"

\section{STUDY SELECTION}

To screen for potential inclusion, the abstracts and, if necessary, complete texts of identified studies were read by one of the authors (G.E.); in cases of uncertainty, the other author (M.H.H.E.) also read the study, with initial discrepancies reconciled by mutual agreement. Studies were included if they enrolled obese patients (body mass $>100 \mathrm{~kg}$ or body mass index [BMI] $>30 \mathrm{~kg} / \mathrm{m}^{2}$ ) or measured anti-Xa concentrations and if they included an LMWH treatment arm; the comparator could be a different dose of LMWH, UFH, or a placebo. Because the number of randomized controlled trials (RCTs) identified in the initial search was low, cohort studies were also included. A 9-step decision-making algorithm was used to review the included studies, to help in determining whether monitoring of LMWH therapy by means of anti-Xa activity in obesity is warranted. ${ }^{25}$ For question 7 of the algorithm, animal studies were included, as there is a paucity of human data suitable for answering this question. Studies that included patients with malignancy, pregnancy, or renal dysfunction were excluded. See Figure 1 for a flow diagram of study selection.

The 9-step decision-making algorithm (see subheadings in the section "Data Synthesis" below) allows clinicians to systematically exercise clinical judgment to ascertain whether monitoring of a particular drug in a particular patient population is warranted. ${ }^{25}$ The algorithm has no scoring system, and while there is no specific rationale for weighting the 9 questions equally, clinical judgment is required to determine in which specific situations monitoring may be warranted. Clearly, the answer to questions 1, 2, 3, and 4 must be "yes" to warrant monitoring. However, the answer to questions 5-9 may be "yes" for certain individuals but "no" for others.

\section{DATA SYNTHESIS}

\section{Is the Patient on the Best Drug for His or Her Specific Disease State and Specific Indication?}

LMWH is approved by Health Canada and the US Food and Drug Administration for prophylaxis of VTE in patients

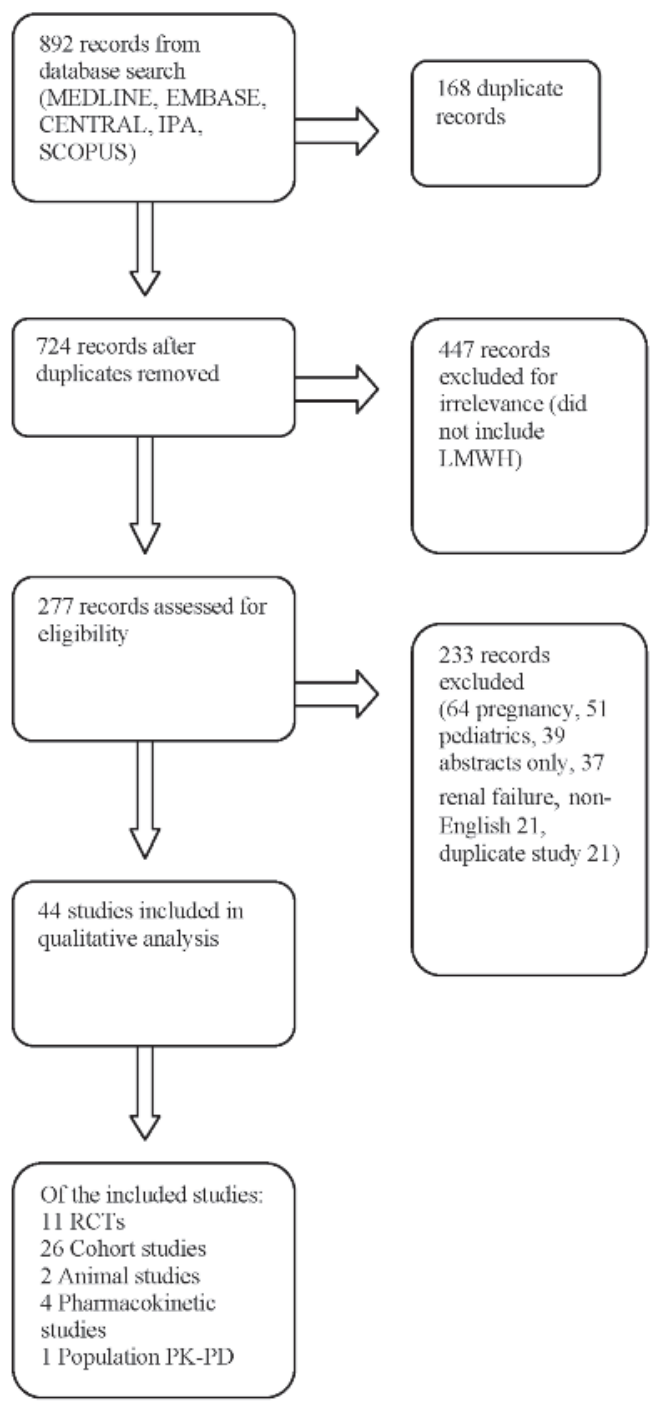

Figure 1. Flow diagram for selection of studies PK-PD = pharmacokinetic-pharmacodynamic, $\mathrm{LMWH}=$ low-molecular-weight heparin, $\mathrm{RCT}=$ randomized controlled trial.

undergoing orthopedic surgery of hip or knee or high-risk abdominal, gynecological, or urological surgery; in patients who are bedridden because of cardiac insufficiency (New York Heart Association Class III or IV heart failure); in patients with acute respiratory failure not requiring ventilator support; and for treatment of deep vein thrombosis with or without pulmonary embolism. ${ }^{17-19,26}$ Enoxaparin and dalteparin are also indicated for treatment of unstable angina, non-Q-wave myocardial infarction, or acute ST-segment elevation myocardial infarction. ${ }^{17-19,26}$ Manufacturer-published dosing guidelines for enoxaparin, dalteparin, and tinzaparin are summarized in Table 1. The American College of Chest Physicians Evidence-Based Clinical Practice Guidelines recommend LMWH for the prevention and treatment of VTE in medical and surgical patients. ${ }^{3}$ The 
Table 1. Manufacturers' Published Dosing Recommendations for Common LMWHs ${ }^{17-19}$

\begin{tabular}{|c|c|c|c|}
\hline LMWH & VTE Prophylaxis & VTE Treatment & Acute Coronary Syndrome \\
\hline Bemiparin & $\begin{array}{l}2500 \text { U SC od } \\
3500 \text { U SC od }\end{array}$ & 5000-10 000 SC od & Not indicated \\
\hline Dalteparin & $\begin{array}{l}2500 \cup \mathrm{SC} \text { od } \\
5000 \cup \mathrm{SC} \text { od }\end{array}$ & $\begin{array}{l}200 \mathrm{U} / \mathrm{kg} \mathrm{SC} \text { od } \\
\text { (maximum } 18000 \mathrm{U} / \text { day) }\end{array}$ & 120 U/kg SC bid \\
\hline Enoxaparin & $\begin{array}{l}30 \mathrm{mg} \mathrm{SC} \text { bid } \\
40 \mathrm{mg} \mathrm{SC} \text { od }\end{array}$ & $\begin{array}{l}1 \mathrm{mg} / \mathrm{kg} \mathrm{SC} \text { bid } \\
1.5 \mathrm{mg} / \mathrm{kg} \mathrm{SC} \text { od }\end{array}$ & $\begin{array}{l}\leq 75 \text { years old: } 30 \mathrm{mg} \mathrm{IV} \\
\text { bolus, then } 1 \mathrm{mg} / \mathrm{kg} \mathrm{SC} \text { bio } \\
>75 \text { years old: } \\
0.75 \mathrm{mg} / \mathrm{kg} \text { bid }\end{array}$ \\
\hline Logiparin & 2500 U SC od & $150 \mathrm{U} / \mathrm{kg} \mathrm{SC}$ od & Not indicated \\
\hline Nadroparin & 2850 U SC od & $171 \mathrm{U} / \mathrm{kg} \mathrm{SC}$ od & Not indicated \\
\hline Parnaparin & $\begin{array}{l}3200 \text { U SC bid } \\
6400 \text { U SC od }\end{array}$ & $\begin{array}{l}6400 \cup \text { SC bid } \\
12800 \text { U SC bid }\end{array}$ & Not indicated \\
\hline Tinzaparin & Not indicated & 175 U/kg SC od & Not indicated \\
\hline
\end{tabular}

bid = twice daily, LMWH = low-molecular-weight heparin, od = once daily, SC = subcutaneous.

VTE $=$ venous thromboembolism

American College of Cardiology Foundation/American Heart Association guideline for managing acute coronary syndromes recommends enoxaparin dosed according to age, weight, and creatinine clearance for up to 8 days or until revascularization. ${ }^{26}$

For VTE prophylaxis in orthopedic surgery, neurosurgery, and trauma, LMWH is preferred over UFH., ${ }^{3,27-30}$ In 2 metaanalyses comparing adjusted-dose UFH with fixed-dose LMWH for treating VTE in medical patients, there were no significant differences in rates of thrombosis or hemorrhagic events. ${ }^{9,10}$ In the treatment of acute coronary syndromes, LMWH has been associated with a reduction in the need for revascularization and is preferred for patients with normal renal function. ${ }^{31,32}$ In patients with VTE and concurrent malignancy, LMWH is superior to UFH and warfarin. ${ }^{33}$ For VTE secondary to pregnancy, LMWH is preferred, as warfarin is teratogenic. ${ }^{3,9,10}$ There are also logistic advantages to LMWH. In cases where outpatient management of VTE is appropriate, LMWH can be used as an anticoagulation bridge to warfarin therapy. ${ }^{3,11}$

The indications outlined here are the same for all patients, regardless of body habitus. ${ }^{17-19,26-33}$

\section{Can the Drug Be Readily Measured in the Desired Biological Matrix?}

The anticoagulant activity of LMWH is monitored by a chromogenic assay in which excess factor $\mathrm{Xa}$ is added to a patient's blood sample, and LMWH-antithrombin III complexes in the blood bind factor Xa and release a chromophore. ${ }^{34-40}$ A standard reagent, developed by the World Health Organization, has been used in contemporary chromogenic assays since $1987 . .^{37,40}$ The inter-laboratory coefficient of variation for this reagent is less than 5\%.38,39 Numerous assay kits are used by clinical laboratories. ${ }^{34,36,37,40}$ Each contains the chromogenic peptide and has a linear range for anti-Xa units from 0.001 to $1.0 \mathrm{U} / \mathrm{mL} .^{41}$ For results recorded as "> $1.0 \mathrm{U} / \mathrm{mL}$ ", the sample is diluted and the assay repeated. ${ }^{17-19,20,40}$ In a study comparing 5 commercially available chromogenic assays for measurement of anti-Xa activity, Kitchen and others ${ }^{41}$ found $43 \%$ interassay variability for enoxaparin and $27 \%$ interassay variability for dalteparin. In another study, Gosselin and others ${ }^{42}$ administered 7 different lots of enoxaparin ( $1 \mathrm{mg} / \mathrm{kg} \mathrm{SC})$ to 20 different patients and assayed samples using the same chromogenic assay. The range of anti-Xa activity was 0.2 to $1.1 \mathrm{U} / \mathrm{mL}$, and there were significant differences in anti-Xa activity between enoxaparin lots $(p<0.01) .{ }^{42}$ Anti-Xa assays available before $1995 \mathrm{did}$ not account for inactivation of factor $\mathrm{Xa}$ by the presence of in vivo plasma calcium and overestimated anti-Xa activity related to LMWH, relative to modern assays. ${ }^{2,14}$ Given the inconsistency in reporting of assay variability, the interpretation of anti-Xa concentrations in clinical practice should be specific to the assay used, and anti-Xa concentrations should be compared only if the same assay is used to obtain every result.

The convention is to measure "peak" anti-Xa concentration about $4 \mathrm{~h}$ after administering an SC dose of LMWH..$^{3-6,17-19}$ Each LMWH preparation has a specific distribution of molecular weight and a specific ratio of anti-Xa to anti-IIa activity. ${ }^{17-19}$ For prophylactic and treatment doses of $\mathrm{LMWH}$, the manufacturers have published mean anti-Xa concentrations measured in healthy volunteers (Table 2). ${ }^{17-19}$ However, the manufacturers have not tested their products in patients weighing more than $120 \mathrm{~kg}$ (enoxaparin), ${ }^{17}$ more than $90 \mathrm{~kg}$ (dalteparin), ${ }^{18}$ or more than $105 \mathrm{~kg}$ (tinzaparin). ${ }^{19}$ There is no specific information in the product monographs on anti-Xa sampling in obesity.

\section{Has a Good Relationship between Drug Concentra- tion and Pharmacological Response Been Reported in Pharmacokinetic Studies Conducted in Humans?}

The relationship between anti-Xa activity and clinical outcome has been studied in numerous clinical trials (Table 3). ${ }^{16,43-54}$ Only 2 trials of LMWH for postoperative VTE prophylaxis showed an association between anti-Xa concentration and clinical 


\section{Table 2. Manufacturers' Published Peak Anti-Factor Xa Concentrations with Various LMWH Regimens}

\begin{tabular}{|c|c|c|c|}
\hline LMWH & $\begin{array}{c}\text { Anti-Xa } 4 \mathrm{~h} \text { after Dose, } \\
\text { Mean } \pm \text { SD (U/mL) }\end{array}$ & $\begin{array}{l}\text { Ratio of Anti-Xa to } \\
\text { Anti-Ila Activity }\end{array}$ & Target Range (U/mL) \\
\hline Enoxaparin $^{17}$ & & $3.7: 1$ & \\
\hline $1 \mathrm{mg} / \mathrm{kg}$ & 0.9 & & $0.6-1.0$ \\
\hline $1.5 \mathrm{mg} / \mathrm{kg}$ & 1.1 & & $>1.0$ \\
\hline Dalteparin $^{18}$ & & $2.8: 1$ & \\
\hline $5000 \mathrm{U}$ & $0.49 \pm 0.13$ & & $<0.6$ \\
\hline $200 \mathrm{U} / \mathrm{kg}$ & $1.2 \pm 0.43$ & & 1.05 \\
\hline Tinzaparin $^{19}$ & & $6: 1$ & \\
\hline $3500 \mathrm{U}$ & 0.15 & & \\
\hline $75 \mathrm{U} / \mathrm{kg}$ & 0.34 & & \\
\hline 150 U/kg & 0.70 & & 0.85 \\
\hline
\end{tabular}

outcome. ${ }^{43,44}$ Koller and others ${ }^{43}$ compared 2 doses of dalteparin (2500 U and $7500 \mathrm{U}$ ) with UFH (5000 U twice daily) in 289 patients and reported more bleeding events with dalteparin 7500 U daily than with UFH ( $47 \%$ versus $10 \% ; p<0.01$ ). Mean peak 4-h anti-Xa concentrations were higher in patients with bleeding than in patients without bleeding $(0.48 \mathrm{U} / \mathrm{mL}$ versus 0.11 $\mathrm{U} / \mathrm{mL}, p<0.01)$. Levine and others ${ }^{44}$ compared postoperative VTE prophylaxis with LMWH (enoxaparin $40 \mathrm{mg}$ daily or $30 \mathrm{mg}$ twice daily) and UFH (5000 U twice daily) in 162 patients undergoing orthopedic surgery. Hemorrhage (as wound hematoma) was more frequent with higher peak anti-Xa concentration obtained $12 \mathrm{~h}$ after administration $(24.5 \%$ with peak anti-Xa $>0.2 \mathrm{U} / \mathrm{mL}$ versus $5.3 \%$ with peak anti-Xa $\leq 0.2 \mathrm{U} / \mathrm{mL}$ ). Conversely, postoperative thrombosis was less frequent with higher trough anti-Xa concentration $(6.3 \%$ with trough anti-Xa $>0.1 \mathrm{U} / \mathrm{mL}$ versus and $14.6 \%$ with trough anti-Xa $\leq 0.1$ $\mathrm{U} / \mathrm{mL}) .{ }^{44}$ The results of these 2 studies suggest a relationship between anti-Xa concentration and clinical outcome.

Seven clinical studies (4 RCTs, 1 subgroup analysis of 5 small RCTs, and 2 cohort trials) measured peak anti-Xa concentrations, obtained about $4 \mathrm{~h}$ after administration, in 2056 patients treated with LMWH for prevention of VTE. ${ }^{45-51}$ Two of the RCTs were performed in an orthopedic surgery setting, one in a general surgery setting, and one in a medicine setting. ${ }^{45-47,51}$ In 2 RCTs, there was no significant difference in anti-Xa concentrations between patients who experienced VTE and those who did not. ${ }^{47,51}$ In the subgroup analysis of 5 RCTs, all of which were performed in an orthopedic surgery setting, there was no association between anti-Xa concentration and hemorrhage or thrombosis. ${ }^{48}$ In a cohort study of 189 elderly medical patients treated with enoxaparin $40 \mathrm{mg}$ SC daily, there were no incidents of VTE and one hemorrhagic event; anti-Xa concentration ranged widely, from 0.2 to $1.1 \mathrm{U} / \mathrm{mL} .^{52} \mathrm{~A}$ population pharmacokinetic-pharmacodynamic model based on data from 96 patients treated with LMWH associated the risk of bruising with age older than 50 years and body weight below $90 \mathrm{~kg}$; there was no association with anti-Xa concentration. ${ }^{53}$
Three studies measured anti-Xa concentrations in patients with VTE who were treated with LMWH. ${ }^{46,47,55}$ One RCT included 194 patients who received either LMWH doses adjusted to anti-Xa concentration $(0.4-0.9 \mathrm{U} / \mathrm{mL})$ or $\mathrm{UFH}$ adjusted to aPTT (1.5 to 3 times baseline). ${ }^{48}$ There was no difference in rate of hemorrhage ( $10 \%$ versus $9 \%$ ), and the mean peak anti-Xa activity on day 3 in patients with bleeding was 0.63 $\mathrm{U} / \mathrm{mL}$ in the LMWH group, which is within the therapeutic range stated by the manufacturer. ${ }^{46}$ In the 2 cohort studies, very few clinical events were reported, and there was no association between anti-Xa concentration and clinical events. ${ }^{49,50}$ Risk of thrombosis and hemorrhage appears not to be predicted by anti-Xa concentration and is perhaps better predicted by the traditional risk factors for bleeding and thrombosis (Table 3). Some reasons for this finding may be the multiple mechanisms by which heparin exerts an anti-thrombotic effect (i.e., by inactivating release of factors IIa, Va, VIIa, and VIIIa and von Willebrand factor and by interacting with endothelial cells and platelets); as such, anti-Xa activity alone may be insufficient to measure clinical effect. ${ }^{2,17-19,47}$

\section{Is the Drug's Pharmacological Response Not Readily Assessable?}

The desired pharmacological effect of LMWH is prevention of thrombosis, and the undesired adverse effect is bleeding. ${ }^{2,4,5}$ Event rates in clinical trials with LMWH treatment groups are about $2 \%$ for thrombosis and $0.5 \%$ for hemorrhage. ${ }^{9,10}$ The symptoms of thrombosis resolve slowly, and the effectiveness of LWMH is not apparent immediately upon initiating therapy. ${ }^{3-5}$ Obesity is an independent risk factor for thrombosis (e.g., increase in procoagulant factors with increasing BMI, additive risk caused by venous stasis, decreased mobility). ${ }^{8,21,56}$ Thus, dosing by total body weight and/or measurement of anti-Xa concentrations to assess therapeutic effect may be advantageous in obese patients. 
Table 3. Summary of Clinical Trials Comparing Anti-Factor Xa Concentration and Clinical Outcome (part 1 of 2)

\begin{tabular}{|c|c|c|c|c|c|c|}
\hline Study & Design & Context & Intervention & Control & $\begin{array}{l}\text { Anti-Xa } \\
\text { Concentration } \\
(\mathrm{U} / \mathrm{mL})^{*}\end{array}$ & Outcome \\
\hline $\begin{array}{l}\text { Koller et al. } \\
(1986)^{43}\end{array}$ & $\begin{array}{l}2 \text { RCTs } \\
(n=289)\end{array}$ & $\begin{array}{l}\text { Elective abdominal } \\
\text { surgery }\end{array}$ & $\begin{array}{l}\text { Dalteparin } \\
7500 \text { U od } \\
\text { Dalteparin } \\
2500 \text { U daily }\end{array}$ & $\begin{array}{l}\text { UFH } 5000 \\
\text { U bid }\end{array}$ & $\begin{array}{l}\text { 4-h peak: } \\
\text { dalteparin } 7500 \mathrm{U}, \\
0.48 \pm 0.12 \\
\text { dalteparin } 2500 \mathrm{U} \\
0.11 \pm 0.05\end{array}$ & $\begin{array}{l}\text { Dalteparin } 7500 \mathrm{U} \text { : discontinue due } \\
\text { to hemorrhage, } 47 \% \text { vs } 10 \% \\
(p<0.01) \\
\text { Dalteparin } 2500 \mathrm{U} \text { : discontinue due } \\
\text { to hemorrhage, } 14.9 \% \text { vs } 15.3 \% \text { (NS) }\end{array}$ \\
\hline $\begin{array}{l}\text { Turpie et al. } \\
(1986)^{45}\end{array}$ & $\begin{array}{l}\text { RCT } \\
(n=100)\end{array}$ & $\begin{array}{l}\text { Elective hip } \\
\text { surgery }\end{array}$ & $\begin{array}{l}\text { Enoxaparin } \\
30 \text { mg bid } x \\
14 \text { days }\end{array}$ & Placebo & $\begin{array}{l}\text { 6-h peak } \\
\text { post-op day } 14: \\
0.20 \pm 0.10\end{array}$ & $\begin{array}{l}\text { Thrombosis: } 12 \% \text { vs } 42 \% \\
(p<0.0007) ; \text { bleeding: } \\
4 \% \text { vs } 4 \% \text { (NS) }\end{array}$ \\
\hline $\begin{array}{l}\text { Bergqvist } \\
\text { et al. } \\
(1986)^{46}\end{array}$ & $\begin{array}{l}\text { RCT } \\
(n=432)\end{array}$ & $\begin{array}{l}\text { Elective } \\
\text { abdominal } \\
\text { surgery }\end{array}$ & $\begin{array}{l}\text { Dalteparin } \\
5000 \text { U od } x \\
5-7 \text { days }\end{array}$ & $\begin{array}{l}\text { UFH } 5000 \\
\text { U bid } \times 5-7 \\
\text { days }\end{array}$ & $\begin{array}{l}\text { 4-h peak, post-op } \\
\text { day 4: LMWH, } \\
0.69 \pm 0.24 ; \text { UFH, } \\
0.11 \pm 0.20\end{array}$ & $\begin{array}{l}\text { Thrombosis: } 4.3 \% \text { vs } 6.4 \% \text { (NS) } \\
\text { Hemorrhage: less with LMWH } \\
\text { (7\% vs } 13 \% \text { wound hematoma; } \\
2 \% \text { vs } 10 \% \text { operation due to bleeding) }\end{array}$ \\
\hline $\begin{array}{l}\text { Levine et al. } \\
(1989)^{44}\end{array}$ & $\begin{array}{l}3 \mathrm{RCTS} \\
(n=162)\end{array}$ & $\begin{array}{l}\text { THR, post-op VTE } \\
\text { prophylaxis }\end{array}$ & $\begin{array}{l}\text { Enoxaparin } \\
40 \text { mg or } \\
60 \text { mg daily }\end{array}$ & $\begin{array}{l}\text { UFH } 5000 \mathrm{U} \\
\text { bid }\end{array}$ & $\begin{array}{l}12 \text { h post-injection } \\
\text { on day } 3\end{array}$ & $\begin{array}{l}\text { Regression analysis: increased risk of } \\
\text { wound hematoma with higher anti-Xa } \\
(>0.2 \mathrm{U} / \mathrm{mL}), 5.3 \% \text { vs } 24.5 \% \\
(p=0.002) \text {; increased risk of } \\
\text { thrombosis with lower anti-Xa } \\
(\leq 0.1 \mathrm{U} / \mathrm{mL}), 6.3 \% \text { vs } 14.6 \% \\
(p=0.03)\end{array}$ \\
\hline $\begin{array}{l}\text { Handeland } \\
\text { et al. } \\
(1990)^{47}\end{array}$ & $\begin{array}{l}2 \text { pro- } \\
\text { spective } \\
\text { cohorts } \\
(n=56)\end{array}$ & $\begin{array}{l}\text { Venographically } \\
\text { proven DVT } \\
\text { (weight } \\
35-100 \mathrm{~kg} \text { ) }\end{array}$ & $\begin{array}{l}\text { Dalteparin } \\
85 \mathrm{U} / \mathrm{kg} \mathrm{SC} \\
\text { q12h, titrated } \\
\text { to anti-Xa } \\
0.5-0.8 \mathrm{U} / \mathrm{mL}\end{array}$ & $\begin{array}{l}\text { UFH IV, } \\
\text { titrated to } \\
\text { anti-Xa } \\
0.3-0.5 \\
\text { U/mL }\end{array}$ & $\begin{array}{l}48 \% \text { LMWH } \\
20 \% \text { UFH within } \\
\text { TR on day } 2\end{array}$ & $\begin{array}{l}3 \text { clinical failures ( } 2 \text { with UFH, } 1 \text { with } \\
\text { LMWH) } \\
\text { No clinically important bleeding }\end{array}$ \\
\hline $\begin{array}{l}\text { Walenga } \\
\text { et al. } \\
(1991)^{16}\end{array}$ & $\begin{array}{l}\text { Post-hoc } \\
\text { analysis of } \\
4 \text { RCTs } \\
(n=\text { NR) }\end{array}$ & $\begin{array}{l}\text { Post-op VTE } \\
\text { prophylaxis }\end{array}$ & $\begin{array}{l}\text { LMWH: } \\
\text { enoxaparin } \\
40 \text { mg od, } \\
\text { fraxiparin } 7500 \\
\text { U od, logiparin } \\
50 \text { U/kg od }\end{array}$ & $\begin{array}{l}\text { UFH } 5000 \\
\text { U bid }\end{array}$ & $\begin{array}{l}\text { 4-h peak, day } 3: \\
\text { enoxaparin, } \\
0.13 \pm 0.08 \\
\text { fraxiparin, } \\
0.11 \pm 0.07 \\
\text { logiparin, } \\
0.31 \pm 0.10\end{array}$ & $\begin{array}{l}\text { No correlation between anti-Xa } \\
\text { and bleeding or thrombosis }\end{array}$ \\
\hline $\begin{array}{l}\text { Nieuwenhuis } \\
\text { et al. } \\
(1991)^{48}\end{array}$ & $\begin{array}{l}\mathrm{RCT} \\
(n=194)\end{array}$ & VTE treatment & $\begin{array}{l}\text { Dalteparin up } \\
\text { to } 10 \text { days, } \\
\text { titrated to } \\
\text { anti-Xa } \\
0.4-0.9 \mathrm{U} / \mathrm{mL}\end{array}$ & $\begin{array}{l}\text { UFH IV up } \\
\text { to } 10 \text { days, } \\
\text { titrated to } \\
\text { anti-Xa } 0.1- \\
0.4 \mathrm{U} / \mathrm{mL}\end{array}$ & $\begin{array}{l}\text { 4-h peak at } \\
\geq 3 \text { days }\end{array}$ & $\begin{array}{l}\text { Total bleeding } 10 \% \\
\text { Bleeding associated with dose per } \\
\text { body surface area, not anti-Xa } \\
\text { concentration } \\
\text { Anti-Xa for patients with bleeding } \\
\text { event: mean } 0.43 \mathrm{U} / \mathrm{mL}\end{array}$ \\
\hline $\begin{array}{l}\text { Bara et al. } \\
(1992)^{49}\end{array}$ & $\begin{array}{l}\text { RCT } \\
(n=1290)\end{array}$ & $\begin{array}{l}\text { General surgery, } \\
\text { post-op VTE } \\
\text { prophylaxis }\end{array}$ & $\begin{array}{l}\text { Logiparin } 2500 \\
\text { or } 3500 \cup \text { od } \\
\times 10 \text { days }\end{array}$ & $\begin{array}{l}\text { UFH } 5000 \\
\cup \text { bid } \times \\
10 \text { days }\end{array}$ & $\begin{array}{l}\text { 4-h peak, day } 3: \\
\text { UFH, } 0.097 \pm \\
0.004 ; \text { logiparin } \\
2500 \cup, 0.152 \pm \\
0.004 ; \text { logiparin } \\
3500 \cup, \\
0.34 \pm 0.003\end{array}$ & $\begin{array}{l}\text { Severe hemorrhage: } 3.3 \% \text { vs } 2.1 \% \text { vs } \\
3.0 \%(p=0.5) \\
\text { No thrombosis with anti-Xa } \\
<0.2 \mathrm{U} / \mathrm{mL} \\
\text { Thrombosis not associated with } \\
\text { anti-Xa (NS: } p=0.15 \text { ) }\end{array}$ \\
\hline $\begin{array}{l}\text { Harenberg } \\
\text { et al. } \\
(1997)^{50}\end{array}$ & $\begin{array}{l}\text { Prospective } \\
\text { cohort } \\
(n=127)\end{array}$ & $\begin{array}{l}\text { VTE treatment } \\
(n=79), \\
\text { heart valve }(n=16), \\
\text { arterial embolism } \\
(n=10), \\
\text { cardiomyopathy } \\
\text { ( } n=15), \text { VTE in } \\
\text { pregnancy }(n=7)\end{array}$ & \multicolumn{2}{|c|}{$\begin{array}{l}\text { Enoxaparin } 2500 \mathrm{U} \text { to } \\
15000 \mathrm{U} \text { per day (average } \\
100 \mathrm{U} / \mathrm{kg} \text { per day); } 2500 \mathrm{U} \\
\text { used in patients with } \\
\text { hepatic cirrhosis }\end{array}$} & $\begin{array}{l}\text { 4-h peak, day 3: } \\
0.1-0.8 \\
\text { (mean } 0.4 \text { ) }\end{array}$ & $\begin{array}{l}3 \text { patients with bleeding } \\
\text { (anti-Xa }=0.15 \mathrm{U} / \mathrm{mL}, 0.32 \mathrm{U} / \mathrm{mL} \text {, } \\
0.41 \mathrm{U} / \mathrm{mL} \text {, respectively) } \\
\text { Recurrent thrombosis NR }\end{array}$ \\
\hline $\begin{array}{l}\text { Kovacs et al. } \\
(1998)^{54}\end{array}$ & $\begin{array}{l}\text { Prospective } \\
\text { cohort } \\
(n=205)\end{array}$ & $\begin{array}{l}\text { Orthopedic } \\
\text { surgery, post-op } \\
\text { VTE prophylaxis }\end{array}$ & \multicolumn{2}{|c|}{ Enoxaparin $30 \mathrm{mg}$ bid } & $\begin{array}{l}\text { 12-h trough, day 5: } \\
\text { VTE, } 0.21 \pm 0.034 ; \\
\text { no VTE, } 0.20 \pm \\
0.008 \text { (NS) }\end{array}$ & 11 patients with VTE \\
\hline
\end{tabular}


Table 3. Summary of Clinical Trials Comparing Anti-Factor Xa Concentration and Clinical Outcome (part 2 of 2)

\begin{tabular}{|c|c|c|c|c|c|c|}
\hline Study & Design & Context & Intervention & Control & $\begin{array}{c}\text { Anti-Xa } \\
\text { Concentration } \\
(\mathrm{U} / \mathrm{mL})^{*}\end{array}$ & Outcome \\
\hline $\begin{array}{l}\text { Bara et al. } \\
(1999)^{51}\end{array}$ & $\begin{array}{l}\mathrm{RCT} \\
(n=440)\end{array}$ & $\begin{array}{l}\text { THR, post-op VTE } \\
\text { prophylaxis } \\
\text { (weight 50-95 kg) }\end{array}$ & $\begin{array}{l}\text { Tinzaparin } \\
4500 \cup \text { od } \\
\times 14 \text { days }\end{array}$ & $\begin{array}{l}\text { Enoxaparin } \\
40 \text { mg od } \\
\times 14 \text { days }\end{array}$ & $\begin{array}{l}\text { 4-h peak, day } 3 \text { : } \\
\text { tinzaparin, } \\
0.38 \pm 0.12 \\
\text { enoxaparin, } \\
0.58 \pm 0.21\end{array}$ & $\begin{array}{l}\text { Venographic DVT } 20 \% \\
\text { Anti-Xa, DVT vs no DVT: with } \\
\text { enoxaparin, } 0.46 \mathrm{U} / \mathrm{mL} \text { vs } 0.39 \mathrm{U} / \mathrm{mL} \\
(p=0.32) ; \text { with tinzaparin, } \\
0.48 \mathrm{U} / \mathrm{mL} \text { vs } 0.54 \mathrm{U} / \mathrm{mL}(p=0.48) \\
\text { Only } 1 \text { case of hemorrhage }\end{array}$ \\
\hline
\end{tabular}

\begin{tabular}{lllll}
\hline Green and & Population & VTE prophylaxis, & Enoxaparin & 2-compart- \\
Duffull & PK-PD & ACS or VTE & $1 \mathrm{mg} / \mathrm{kg}$ bid or & ment \\
$(2003)^{53}$ & model & treatment & $40 \mathrm{mg}$ od; & first-order \\
& $(n=96)$ & & anti-Xa & input \\
& & & concentration \\
& & measured in & \\
& & & \\
& & &
\end{tabular}

Bruising incidence used as outcome for pharmacodynamic model; age $>75$ years, $\mathrm{CrCl}<60 \mathrm{~mL} / \mathrm{min}$, and female sex associated with increased risk of bruising

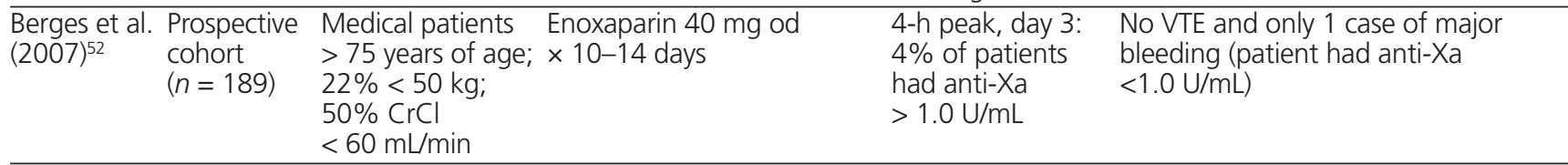

$\overline{\mathrm{ACS}}=$ acute coronary syndrome, anti-Xa = anti-factor Xa, bid = twice daily, $\mathrm{CrCl}=$ creatinine clearance, DVT = deep-vein thrombosis, IV = intravenous, LMWH = low-molecular-weight heparin, NR = not reported, NS = not significant, od = once daily, PK-PD = pharmacokinetic-pharmacodynamic, RCT = randomized control trial, SC = subcutaneous, THR = total hip replacement, $\mathrm{TR}=$ therapeutic range, UFH = unfractionated heparin, VTE = venous thromboembolism .

*Mean \pm standard deviation.

\section{Does the Relationship between Concentration and Pharmacological Response Still Apply to the Specific Disease State and Indication in Patients with Obesity?}

Clinical studies of LWMH for prevention and treatment of VTE that included obese patients were reviewed. In bariatric surgery, LMWH for postoperative VTE prophylaxis has been studied in 5 cohort studies and 1 open-label RCT with 1566 patients. ${ }^{57-62}$ Four studies involved enoxaparin and 1 each dalteparin and parnaparin. The overall mean BMI was $50 \mathrm{~kg} / \mathrm{m}^{2}$ and mean body weight was $150 \mathrm{~kg}$, with the heaviest patient weighing $185 \mathrm{~kg} .{ }^{57-62}$ In general, LMWH dose was fixed (i.e., all patients received the same dose regardless of body weight), and anti-Xa concentration was significantly lower in patients with greater body weight. ${ }^{57-62}$ Scholten and others ${ }^{57}$ (in a study with 481 patients, mean BMI $51 \mathrm{~kg} / \mathrm{m}^{2}$ ) reported no statistically significant difference in risk of thrombosis with enoxaparin $30 \mathrm{mg}$ twice daily or $40 \mathrm{mg}$ twice daily; however, fewer patients in the 40-mg group experienced thrombosis ( 5 events versus 2 events; $p=0.1$ ). This study enrolled consecutive patients, with the $40-\mathrm{mg}$ group being treated subsequent to the $30-\mathrm{mg}$ group and having shorter surgical time and shorter duration of hospital stay, which would have reduced the risk of VTE and potentially biased the risk of thrombosis in favour of the 40-mg group. ${ }^{57} \mathrm{In}$ an open-label RCT (66 patients, BMI $>36 \mathrm{~kg} / \mathrm{m}^{2}$ ), fixed-dose parnaparin produced anti-Xa concentrations that were propor- tional to total body weight, and no thrombosis or hemorrhage was reported..$^{58}$ In a large cohort study of enoxaparin $40 \mathrm{mg}$ SC twice daily (more than 600 obese patients, mean BMI $47 \mathrm{~kg} / \mathrm{m}^{2}$ ), only 3 symptomatic thrombotic events and no hemorrhagic events were reported. ${ }^{60}$ Anti-Xa concentrations could be predicted from total body weight and daily dose (Table 4). ${ }^{57-62}$

Four studies in patients undergoing general and orthopedic surgery with logiparin and 3 cohort studies with enoxaparin and bemiparin were reviewed. ${ }^{63-69}$ Obesity was defined as BMI greater than $30 \mathrm{~kg} / \mathrm{m}^{2}$ and body weight greater than $100 \mathrm{~kg}$. Samama and others, ${ }^{66}$ in a study involving 817 patients, observed a significantly increased risk of venographically detected VTE in obese patients (BMI $>32 \mathrm{~kg} / \mathrm{m}^{2}$ ) relative to non-obese patients when treated with enoxaparin $40 \mathrm{mg} /$ day, but these authors did not report anti-Xa concentrations. A study of enoxaparin $30 \mathrm{mg}$ twice daily in 205 patients showed no significant difference in anti-Xa concentration between patients who developed VTE and those who did not. ${ }^{64}$ In the only study with fixed-dose logiparin (total of 1290 patients, 340 of whom were obese), anti-Xa concentration was negatively correlated with total body weight. ${ }^{63}$ In general, there was increased risk for venographically detected VTE in obese patients treated with fixed-dose LMWH and no increased risk of a hemorrhagic event in obese patients treated with higher doses of LMWH. Overall, in studies reporting anti-Xa concentration, it was not associated with risk of VTE or hemorrhage, as summarized in Table 5.63-66 
Table 4. Summary of Clinical Studies in Bariatric Surgery that Included Obese Patients

\begin{tabular}{|c|c|c|c|c|c|c|}
\hline Study & Design & Participant Weight & Intervention & Comparison & $\begin{array}{l}\text { Anti-Xa } \\
\text { Concentration } \\
(\mathrm{U} / \mathrm{mL})^{*}\end{array}$ & Outcome \\
\hline $\begin{array}{l}\text { Scholten } \\
\text { et al. } \\
(2002)^{57}\end{array}$ & $\begin{array}{l}\text { Retrospective } \\
\text { cohort } \\
(n=481)\end{array}$ & $\begin{array}{l}\text { Mean BMl } \\
51 \mathrm{~kg} / \mathrm{m}^{2}\end{array}$ & $\begin{array}{l}\text { Group l: } \\
\text { enoxaparin } 30 \mathrm{mg} \\
\text { bid }(n=92) \\
\text { Group II: } \\
\text { enoxaparin } \\
40 \text { mg bid } \\
(n=389)\end{array}$ & NR & NR & $\begin{array}{l}7 \text { VTE (1.4\%); } 2 \text { in group I } \\
\text { and } 5 \text { in group II } \\
2 \text { bleeding events } \\
\text { ( } 1 \text { in each group) }\end{array}$ \\
\hline $\begin{array}{l}\text { Borkgren- } \\
\text { Okonek } \\
\text { et al. } \\
(2008)^{59}\end{array}$ & $\begin{array}{l}\text { Prospective, } \\
\text { open-label } \\
(n=223)\end{array}$ & $\begin{array}{l}\text { Mean BMI } \\
50.4 \mathrm{~kg} / \mathrm{m}^{2}\end{array}$ & $\begin{array}{l}\text { Enoxaparin } \\
40 \mathrm{mg} \text { bid if } \\
\mathrm{BMl} \leq 50 \mathrm{~kg} / \mathrm{m}^{2} \\
+/- \text { mechanical } \\
\text { prophylaxis at } \\
\text { discretion of } \\
\text { clinician }\end{array}$ & $\begin{array}{l}\text { Enoxaparin } \\
60 \mathrm{mg} \text { bid if } \\
\text { BMl > } 50 \mathrm{~kg} / \mathrm{m}^{2} \\
+/- \text { mechanical } \\
\text { prophylaxis } \\
\text { at discretion } \\
\text { of clinician }\end{array}$ & $\begin{array}{l}\text { 4-h peak concs: } \\
\text { BMl } \leq 50 \mathrm{~kg} / \mathrm{m}^{2} \\
0.32 \pm 0.10 \\
\text { BMI > } 50 \mathrm{~kg} / \mathrm{m}^{2} \\
0.26 \pm 0.13 \text { (NS) }\end{array}$ & $\begin{array}{l}1 \text { case of nonfatal VTE } \\
\text { (rate } 0.45 \% \text { ) } \\
\text { and } 3 \text { cases of major } \\
\text { bleeding (rate } 1.79 \% \text { ) }\end{array}$ \\
\hline $\begin{array}{l}\text { Escalante- } \\
\text { Tattersfield } \\
\text { et al. } \\
(2008)^{60}\end{array}$ & $\begin{array}{l}\text { Retrospective } \\
\text { cohort } \\
(n=618)\end{array}$ & $\begin{array}{l}\text { Post-op Roux- } \\
\text { en-Y surgery; } \\
\text { mean weight } \\
140 \mathrm{~kg} \text {, mean } \\
\text { BMl } 47 \mathrm{~kg} / \mathrm{m}^{2}\end{array}$ & $\begin{array}{l}\text { UFH } 5000 \text { U SC q8 } \\
\text { enoxaparin } 40 \mathrm{mg} \\
\text { sequential compres } \\
\text { until ambulation }\end{array}$ & $\begin{array}{l}3 \mathrm{~h} \times 24 \mathrm{~h} \text {, then } \\
\mathrm{SC} \text { bid with } \\
\text { ssion device }\end{array}$ & NR & $\begin{array}{l}1 \text { case of asymptomatic } \\
\text { VTE, no hemorrhagic events }\end{array}$ \\
\hline $\begin{array}{l}\text { Simone et al. } \\
(2008)^{62}\end{array}$ & $\begin{array}{l}\text { Prospective } \\
\text { cohort } \\
(n=40)\end{array}$ & & $\begin{array}{l}\text { Enoxaparin } \\
60 \mathrm{mg} \text { bid }\end{array}$ & $\begin{array}{l}\text { Enoxaparin } \\
40 \mathrm{mg} \text { bid }\end{array}$ & $\begin{array}{l}\text { Non-obese } 0.21 \\
\text { vs obese } 0.43 \\
(p<0.001)\end{array}$ & $\begin{array}{l}\text { Only } 1 \text { hemorrhagic event } \\
\text { reported }\end{array}$ \\
\hline $\begin{array}{l}\text { Imberti et al. } \\
(2009)^{58}\end{array}$ & $\begin{array}{l}\text { Open-label, } \\
\text { randomized, } \\
\text { consecutive } \\
\text { patients } \\
(n=66)\end{array}$ & $\begin{array}{l}\text { Post-op; BMl } \\
>36 \mathrm{~kg} / \mathrm{m}^{2}\end{array}$ & $\begin{array}{l}\text { Parnaparin } \\
4250 \text { U/day }\end{array}$ & $\begin{array}{l}\text { Parnaparin } \\
6400 \text { U/day }\end{array}$ & $\begin{array}{l}\text { 4-h peak, day 6: } \\
\text { parnaparin } 4250 \\
\text { U/day, } 0.18 \\
(0.13-0.25) ; \\
\text { parnaparin } 6400 \\
\text { U/day, } 0.41 \\
(0.32-0.51)\end{array}$ & $\begin{array}{l}\text { No thrombosis or } \\
\text { hemorrhage } \\
\text { Anti-Xa for patients } \\
\geq 45 \mathrm{~kg} / \mathrm{m}^{2} \text { vs } \\
<45 \mathrm{~kg} / \mathrm{m}^{2} \text { (NS) }\end{array}$ \\
\hline $\begin{array}{l}\text { Simoneau } \\
\text { et al. } \\
(2010)^{61}\end{array}$ & $\begin{array}{l}\text { Retrospective } \\
\text { cohort } \\
(n=135)\end{array}$ & $\begin{array}{l}\text { Mean body } \\
\text { weight } 148 \text { kg; } \\
\text { mean BMl } \\
52 \mathrm{~kg} / \mathrm{m}^{2}\end{array}$ & \multicolumn{2}{|c|}{ Dalteparin 7500 U SC od } & $\begin{array}{l}\text { TR 0.2-0.5; mean } \\
\text { body weight of } \\
\text { subgroups based } \\
\text { on anti-Xa on } \\
\text { day } 4:<0.2 \\
160 \mathrm{~kg} ; 0.2-0.5, \\
145 \mathrm{~kg} ;>0.5 \\
136 \mathrm{~kg}(p<0.01)\end{array}$ & $\begin{array}{l}\text { No thrombotic or } \\
\text { hemorrhagic events } \\
\text { reported }\end{array}$ \\
\hline
\end{tabular}

Anti-Xa = anti-factor Xa, bid = twice daily, BMI = body mass index, concs = concentrations, NR = not reported, NS = not significant, od = once daily, SC = subcutaneous, TR = therapeutic range, UFH = unfractionated heparin, VTE = venous thromboembolism.

*Mean (range) or mean \pm standard deviation, unless otherwise reported.

In an RCT of general medical patients (total of 3706 patients, 1112 of whom were obese) treated with dalteparin 5000 U SC once daily, the rate of VTE was not significantly different between obese and non-obese patients. ${ }^{67}$ In 3 cohort studies of enoxaparin (total of 171 patients, 82 of whom were obese) that used weight-based dosing according to total body weight, up to $90 \%$ of patients were within the target anti-Xa range recommended in the product monograph. ${ }^{68-70}$ Dosing enoxaparin 40 $\mathrm{mg}$ or $60 \mathrm{mg}$ daily at a fixed dose or on the basis of body weight $(0.5 \mathrm{mg} / \mathrm{kg}$ daily) produced predictable anti-Xa concentrations. The results of these studies, summarized in Table 6, 67-70 indicate that for prophylaxis of VTE in surgical or medical patients, anti-Xa concentration is proportional to total body weight, and a larger dose of LMWH is justified in obese patients. Anti$\mathrm{Xa}$ levels are not associated with risk of thrombosis or hemorrhage.
The search yielded 7 studies of LMWH given for treatment of VTE or acute coronary syndromes. ${ }^{71-77}$ For VTE, one subgroup of an $\mathrm{RCT}^{73}$ and 5 cohort studies ${ }^{71,72,74-76}$ included obese patients ( $>100 \mathrm{~kg}$ or $\left.>30 \mathrm{~kg} / \mathrm{m}^{2}\right)$. In the MATISSE RCT for treatment of VTE (total of 2217 patients, 496 of whom were obese), enoxaparin $1 \mathrm{mg} / \mathrm{kg}$ twice daily was used for all patients, with the dose based on total body weight. ${ }^{73}$ Anti-Xa concentrations were not reported, and there was no significant difference in rates of VTE or hemorrhage between obese and non-obese patients. In the cohort studies reporting anti-Xa concentrations, there was no significant difference in anti-Xa concentration or in thrombosis or hemorrhage between obese and non-obese patients when the dose was based on total body weight. ${ }^{71,72,74-76}$ In the subgroup analysis of the ESSENCE and TIMI-11B RCTs of enoxaparin versus UFH in acute coronary syndrome (total of 3171 obese patients), there was no signifi- 
Table 5. Summary of Clinical Studies in Orthopedic and General Surgery that Included Obese Patients

\begin{tabular}{|c|c|c|c|c|c|c|}
\hline Study & Design & Context & Intervention & Comparison & $\begin{array}{l}\text { Anti-Xa } \\
\text { Concentration } \\
(\mathrm{U} / \mathrm{mL})^{*}\end{array}$ & Outcome \\
\hline $\begin{array}{l}\text { Leizorovicz } \\
\text { et al. } \\
(1993)^{63}\end{array}$ & $\begin{array}{l}\text { RCT } \\
(n=1290)\end{array}$ & $\begin{array}{l}\text { Post-op VTE } \\
\text { prophylaxis }\end{array}$ & $\begin{array}{l}\text { Logiparin } 2500 \mathrm{U} \\
\text { od or } 3500 \mathrm{U} \text { od }\end{array}$ & UFH 5000 U bid & $\begin{array}{l}\text { Anti-Xa correlated } \\
\text { with body weight }\end{array}$ & NS for clinical outcome \\
\hline $\begin{array}{l}\text { Samama } \\
\text { et al. } \\
(1995)^{66}\end{array}$ & $\begin{array}{l}\text { Retrospective } \\
\text { analysis } \\
(n=817)\end{array}$ & $\begin{array}{l}\text { Orthopedic } \\
\text { surgery, VTE } \\
\text { prophylaxis }\end{array}$ & $\begin{array}{l}\text { Enoxaparin } 40 \mathrm{mg} \\
\text { Obese (BMl > } 32 \mathrm{k}\end{array}$ & $\begin{array}{l}\text { od } \\
\left.\mathrm{g} / \mathrm{m}^{2}\right) \text { vs non-obese }\end{array}$ & NR & $\begin{array}{l}\text { Venographically detected VTE } \\
31.8 \% \text { vs } 16.7 \% \\
(p<0.0001)\end{array}$ \\
\hline $\begin{array}{l}\text { Kovacs et al. } \\
(1998)^{64}\end{array}$ & $\begin{array}{l}\text { Prospective } \\
\text { cohort } \\
(n=205)\end{array}$ & $\begin{array}{l}\text { Orthopedic } \\
\text { surgery, VTE } \\
\text { prophylaxis }\end{array}$ & Enoxaparin $30 \mathrm{mg}$ & bid & & $\begin{array}{l}\text { 12-h trough, day 5: VTE } \\
(n=11), 0.21 \pm 0.034 \\
\text { No VTE }(n=194) \\
0.20 \pm 0.008 \text { (NS) }\end{array}$ \\
\hline $\begin{array}{l}\text { Vavken et al. } \\
(2009)^{65}\end{array}$ & $\begin{array}{l}\text { Prospective } \\
\text { cohort } \\
(n=723)\end{array}$ & $\begin{array}{l}\text { Orthopedic } \\
\text { surgery, VTE } \\
\text { prophylaxis } \\
\text { (weight > } 90 \mathrm{~kg} \text { ) }\end{array}$ & $\begin{array}{l}\text { Bemiparin } \\
5000 \text { U od }\end{array}$ & $\begin{array}{l}\text { Bemiparin } \\
3500 \text { U od }\end{array}$ & NR & $\begin{array}{l}\text { Bleeding: bemiparin } 5000 \mathrm{U}, \\
1 \text { event vs bemiparin } 3500 \mathrm{U} \text {, } \\
2 \text { events (NS) } \\
\text { Symptomatic VTE: bemiparin } \\
3500 \mathrm{U}, 0.392 / \mathrm{PY} \text { vs } \\
\text { bemiparin } 5000 \mathrm{U}, 0.09 / \mathrm{PY}\end{array}$ \\
\hline
\end{tabular}

Anti-Xa = anti-factor Xa, bid = twice daily, NR = not reported, NS = not significant, od = once daily, PY = person-year, $\mathrm{RCT}=$ randomized control trial, VTE = venous thromboembolism, UFH = unfractionated heparin.

Table 6. Summary of Clinical Studies for VTE Prophylaxis in Medical Patients, Including Obese Patients

\begin{tabular}{|c|c|c|c|c|c|}
\hline Study & Design & Participants & Intervention & $\begin{array}{l}\text { Anti-Xa } \\
\text { Concentration } \\
(\mathrm{U} / \mathrm{mL})^{*}\end{array}$ & Outcome \\
\hline $\begin{array}{l}\text { Kucher } \\
\text { et al. } \\
(2005)^{67}\end{array}$ & $\begin{array}{l}\text { Subgroup } \\
\text { analysis of } \\
\text { PREVENT } \\
\text { RCT } \\
(n=3706)\end{array}$ & $\begin{array}{l}30 \% \text { were obese } \\
\left(>30 \mathrm{~kg} / \mathrm{m}^{2}\right)\end{array}$ & $\begin{array}{l}\text { Dalteparin } \\
5000 \text { U od }\end{array}$ & NR & $\begin{array}{l}\text { Symptomatic VTE } \\
\text { obese ( } 2.8 \% \text { vs } 4.3 \% \text {; NS) }\end{array}$ \\
\hline $\begin{array}{l}\text { Jiménez } \\
\text { et al. } \\
(2008)^{68}\end{array}$ & $\begin{array}{l}\text { Prospective } \\
\text { cohort } \\
(n=112)\end{array}$ & $\begin{array}{l}\text { Medical patients, } \\
21 \% \text { obese }\end{array}$ & $\begin{array}{l}\text { Enoxaparin } 40 \mathrm{mg} \text { od } \times 7 \text { days } \\
\text { (mean duration of therapy) }\end{array}$ & $\begin{array}{l}\text { 4-h peak, day 3: } \\
\text { BMl < 23 kg/m², } \\
0.28 \pm 0.23 ; \\
\text { BMI } 23-26 \mathrm{~kg} / \mathrm{m}^{2} \\
0.23 \pm 0.35 ; \\
\text { BMl } 26-29 \mathrm{~kg} / \mathrm{m}^{2} \\
0.15 \pm 0.09 ; \\
\text { BMI > } 29 \mathrm{~kg} / \mathrm{m}^{2} \\
0.13 \pm 0.11\end{array}$ & $\begin{array}{l}\text { No cases of major bleeding, } \\
2 \text { cases of proximal DVT } \\
\text { with anti-Xa }<0.10 \mathrm{U} / \mathrm{mL}\end{array}$ \\
\hline $\begin{array}{l}\text { Rondina } \\
\text { et al. } \\
(2010)^{69}\end{array}$ & $\begin{array}{l}\text { Prospective } \\
\text { cohort } \\
(n=28)\end{array}$ & $\begin{array}{l}\text { Medical patients, } \\
\text { BMl > } 35 \mathrm{~kg} / \mathrm{m}^{2}\end{array}$ & $\begin{array}{l}\text { Mean enoxaparin dose } 67 \mathrm{mg} / \text { day; } \\
\text { mean BMl } 48 \mathrm{~kg} / \mathrm{m}^{2} \text {; mean } \\
\text { weight } 136 \mathrm{~kg}\end{array}$ & $\begin{array}{l}\text { 4-h peak: } \\
0.25 \pm 0.11\end{array}$ & $\begin{array}{l}\text { No bleeding or hemorrhagic } \\
\text { events }\end{array}$ \\
\hline $\begin{array}{l}\text { Freeman } \\
\text { et al. } \\
(2012)^{70}\end{array}$ & $\begin{array}{l}\text { Prospective } \\
\text { cohort } \\
(n=31)\end{array}$ & $\begin{array}{l}\text { Prophylaxis, } \\
\text { mean weight } \\
150 \mathrm{~kg}\end{array}$ & $\begin{array}{l}\text { Enoxaparin: } 40 \mathrm{mg} \text { od or } 0.4 \mathrm{mg} / \mathrm{kg} \\
\text { od or } 0.5 \mathrm{mg} / \mathrm{kg} \text { od }\end{array}$ & $\begin{array}{l}\text { 4-h peak target } \\
\text { anti-Xa } \\
0.2-0.5 \mathrm{U} / \mathrm{mL}\end{array}$ & $\begin{array}{l}\text { Achievement of target } \\
\text { anti-Xa: } 90 \% \text { with } \\
0.5 \mathrm{mg} / \mathrm{kg} \text { od, } 38 \% \text { with } \\
0.4 \mathrm{mg} / \mathrm{kg} \text { od, } 20 \% \text { with } \\
40 \mathrm{mg} / \mathrm{day}(p<0.001) \\
\text { No bleeding or hemorrhagic } \\
\text { events }\end{array}$ \\
\hline
\end{tabular}

Anti-Xa = anti-factor Xa, BMI = body mass index, DVT = deep vein thrombosis, NS = not significant, od = once daily,

$\mathrm{RCT}=$ randomized control trial, $\mathrm{VTE}=$ venous thromboembolism .

*Mean \pm standard deviation, unless otherwise reported.

cant difference in death, myocardial infarction, or stroke between obese patients $\left(>30 \mathrm{~kg} / \mathrm{m}^{2}\right.$ ) and normal-weight patients. ${ }^{72}$ Dosing of LMWH (dalteparin and enoxaparin) by total body weight in obese patients (generally $>100 \mathrm{~kg}$ [maximum $188 \mathrm{~kg}$ ] and $>30 \mathrm{~kg} / \mathrm{m}^{2}$ ) produced predictable anti-Xa concentrations that were not significantly different between patients who did and did not experience recurrent thrombosis or hemorrhage (Table 7). ${ }^{71-77}$ Treatment of VTE with LMWH dosing based on total body weight produces predictable anti$\mathrm{Xa}$ levels even in obese patients and, in studies that did measure anti-Xa levels, no association with thrombosis or hemorrhage was apparent. 
Table 7. Summary of Clinical Studies of VTE Treatment that Included Obese Patients

\begin{tabular}{|c|c|c|c|c|c|c|}
\hline Study & Design & $\begin{array}{l}\text { Context and } \\
\text { Participants }\end{array}$ & Intervention & Comparison & $\begin{array}{l}\text { Anti-Xa } \\
\text { Concentration } \\
(\mathrm{U} / \mathrm{mL})^{*}\end{array}$ & Outcome \\
\hline $\begin{array}{l}\text { Wilson et al. } \\
(2001)^{71}\end{array}$ & $\begin{array}{l}\text { Prospective } \\
\text { cohort } \\
\text { anticoagu- } \\
\text { lation } \\
\text { bridging } \\
(n=37)\end{array}$ & $\begin{array}{l}\text { Group A, within } \\
20 \% \text { of IBW; } \\
\text { Group B, within } \\
20 \%-40 \% \text { of } \\
\text { IBW; Group C, } \\
>40 \% \text { IBW }\end{array}$ & \multicolumn{2}{|c|}{$\begin{array}{l}\text { Dalteparin } 200 \mathrm{U} / \mathrm{kg} \text { SC od dosed by } \\
\text { TBW } \times 5 \text { days }\end{array}$} & $\begin{array}{l}\text { 4-h peak, day 3: } \\
\text { Grp A, } 1.01 \pm 0.20 ; \\
\text { Grp B, } 0.97 \pm 0.21 \\
\text { Grp C, } 1.12 \pm 0.22 \\
(p>0.2)\end{array}$ & $\begin{array}{l}\text { No thromboembolic or } \\
\text { hemorrhagic events } \\
\text { occurred at 90-day follow up }\end{array}$ \\
\hline $\begin{array}{l}\text { Spinler et al. } \\
(2003)^{72}\end{array}$ & $\begin{array}{l}\text { Subgroup } \\
\text { analysis } \\
\text { from } \\
\text { ESSENCE } \\
\text { and TIMI } \\
11 \mathrm{~B} \\
(n=3171)\end{array}$ & $\begin{array}{l}\text { Obese patients } \\
\left(\text { BMI > } 30 \mathrm{~kg} / \mathrm{m}^{2}\right) \\
\text { with NSTE ACS }\end{array}$ & $\begin{array}{l}\text { Enoxaparin } \\
1 \mathrm{mg} / \mathrm{kg} \text { bid }\end{array}$ & UFH IV & NR & $\begin{array}{l}\text { Death, Ml, or } \\
\text { revascularization } \\
\text { OR 0.78 ( } 95 \% \text { Cl 0.61-1.0) } \\
\text { Any bleeding OR 2.42 } \\
(95 \% \text { Cl 0.69-3.45) }\end{array}$ \\
\hline $\begin{array}{l}\text { Smith and } \\
\text { Canton } \\
(2003)^{74}\end{array}$ & $\begin{array}{l}\text { Prospective } \\
\text { cohort } \\
(n=21)\end{array}$ & $\begin{array}{l}\text { VTE bridging, } \\
\text { mean weight } \\
118 \mathrm{~kg}\end{array}$ & \multicolumn{2}{|c|}{$\begin{array}{l}\text { Dalteparin: } 200 \mathrm{U} / \mathrm{kg} \text { od or } \\
120 \mathrm{U} / \mathrm{kg} \text { bid }\end{array}$} & $\begin{array}{l}\text { 4-h peak, day } 3: \\
0.9 \pm 0.11 \\
1.1 \pm 0.23\end{array}$ & $\begin{array}{l}\text { No correlation between body } \\
\text { weight and anti-Xa } \\
(r=-0.24)\end{array}$ \\
\hline $\begin{array}{l}\text { Al-Yaseen } \\
\text { et al. } \\
(2005)^{75} \\
\end{array}$ & $\begin{array}{l}\text { Retrospective } \\
\text { chart review } \\
(n=193)\end{array}$ & $\begin{array}{l}\text { VTE treatment, } \\
\text { weight }>90 \mathrm{~kg} \\
\text { (mean } 114 \mathrm{~kg})\end{array}$ & $\begin{array}{l}\text { Dalteparin } \\
200 \mathrm{U} / \mathrm{kg} \text { OD }\end{array}$ & $\begin{array}{l}\text { Dalteparin } \\
100 \mathrm{U} / \mathrm{kg} \text { bid }\end{array}$ & $\mathrm{NR}$ & $\begin{array}{l}1 \text { case of major bleeding in } \\
\text { each group }\end{array}$ \\
\hline $\begin{array}{l}\text { Bazinet et al. } \\
(2005)^{76}\end{array}$ & $\begin{array}{l}\text { Prospective } \\
\text { cohort } \\
(n=51)\end{array}$ & $\begin{array}{l}\text { VTE treatment } \\
\left(\mathrm{BMI}>30 \mathrm{~kg} / \mathrm{m}^{2}\right)\end{array}$ & $\begin{array}{l}\text { Enoxaparin } \\
1.5 \mathrm{mg} / \mathrm{kg} \text { od }\end{array}$ & $\begin{array}{l}\text { Enoxaparin } \\
1 \mathrm{mg} / \mathrm{kg} \text { bid }\end{array}$ & $\begin{array}{l}\text { 4-h peak, day } 3: \\
1.5 \mathrm{mg} / \mathrm{kg}, \\
1.15(1.02-1.28) ; \\
1 \mathrm{mg} / \mathrm{kg} \\
1.17(1.08-1.25)\end{array}$ & NS by body weight \\
\hline $\begin{array}{l}\text { Barba et al. } \\
(2005)^{77}\end{array}$ & $\begin{array}{l}\text { RIETE } \\
\text { registry } \\
(n=8845)\end{array}$ & $\begin{array}{l}\text { Consecutive } \\
\text { patients with VTE } \\
(<50 \mathrm{~kg}, \\
50-100 \mathrm{~kg} \text {, } \\
>100 \mathrm{~kg}) ; \\
\text { 3-month follow-up }\end{array}$ & \multicolumn{2}{|l|}{$\begin{array}{l}\text { 95\% LMWH } \\
5 \% \text { UFH } \\
\text { Long-term wa } \\
\text { Long-term LM }\end{array}$} & NR & $\begin{array}{l}\text { Recurrent VTE NS across } \\
\text { weight categories } \\
\text { All bleeds OR } 2.2(95 \% \mathrm{Cl} \\
1.2-4.0) \text { in patients < } 50 \mathrm{~kg} \text {; } \\
\text { mortality OR } 2.7(95 \% \mathrm{Cl} \\
1.5-4.7)\end{array}$ \\
\hline $\begin{array}{l}\text { Davidson } \\
\text { et al. } \\
(2007)^{73}\end{array}$ & $\begin{array}{l}\text { Subgroup } \\
\text { analysis } \\
\text { from } \\
\text { MATISSE } \\
\text { RCT (VTE } \\
\text { treatment) } \\
(n=2217) \\
\end{array}$ & $\begin{array}{l}\text { Weight > } 100 \mathrm{~kg} \\
\mathrm{BMI}>30 \mathrm{~kg} / \mathrm{m}^{2}\end{array}$ & $\begin{array}{l}\text { Fondaparinux } \\
5 \mathrm{mg}(<50 \mathrm{~kg}), \\
7.5 \mathrm{mg} \\
(50-100 \mathrm{~kg}) \\
10 \mathrm{mg}(>100 \mathrm{~kg})\end{array}$ & $\begin{array}{l}\text { Enoxaparin } \\
1 \mathrm{mg} / \mathrm{kg} \text { bid }\end{array}$ & NR & $\begin{array}{l}\text { VTE and hemorrhage: } \\
\text { weight }<100 \mathrm{~kg} \text { vs weight } \\
>100 \mathrm{~kg} \text { (NS) }\end{array}$ \\
\hline
\end{tabular}

Anti-Xa = anti-factor $\mathrm{Xa}$, bid = twice daily, $\mathrm{BMI}=$ body mass index, $\mathrm{Cl}=$ confidence interval, IBW = ideal body weight,

IV = intravenous, LMWH = low-molecular-weight heparin, $\mathrm{MI}=$ myocardial infarction, NR = not reported, NS = not significant,

NSTE ACS = non-ST segment elevation acute coronary syndrome, od = once daily, OR = odds ratio, $r=$ correlation coefficient,

$\mathrm{RCT}=$ randomized controlled trial, SC = subcutaneous, TBW = total body weight, UFH = unfractionated heparin,

VTE $=$ venous thromboembolism.

* Mean \pm standard deviation or mean (range), unless otherwise reported.

No studies were identified that attempted to distinguish the impact of confounders (e.g., comorbidities such as diabetes mellitus, cardiovascular disease, or cancer) on the predictability of pharmacokinetic parameters in obesity.

\section{Does the Drug Have a Narrow Therapeutic Range for the Specific Disease State and Indication in Patients with Obesity?}

A meta-analysis of RCTs for treatment of VTE with LMWH generated a relatively low estimated risk of bleeding with weight-based dosing (i.e., 1\%). ${ }^{9}$ Each LMWH preparation has a specific therapeutic range in terms of anti-Xa concentration (Table 2), and there are no specific data on the therapeutic range of LMWH in obese patients. Thus, the following discussion pertains to all patients treated with LMWH. In clinical studies, the risk of bleeding with LMWH has been associated with increased total dose, which suggests a predictable dose-response relationship between LMWH and bleeding. ${ }^{17-19,43,44}$ In animal models, the lower limit of the lethal range for enoxaparin is 160 $\mathrm{mg} / \mathrm{kg} .{ }^{17}$ The lethal dose of dalteparin has not been determined, and mice that were given $100000 \mathrm{U} / \mathrm{kg}$ survived. ${ }^{18}$ No lethal dose has been determined for tinzaparin, but rats given $62500 \mathrm{U} / \mathrm{kg}$ daily for at least 6 months developed osteopenia. ${ }^{19}$ In one case, a patient received $72000 \mathrm{U}$ of dalteparin and had a random anti-Xa concentration of $6.2 \mathrm{U} / \mathrm{mL}$ at $7 \mathrm{~h}$ after injection; hemorrhage did not occur. ${ }^{78} \mathrm{LMWH}$ is associated with a low risk 
of bleeding in clinical studies, and there is an apparent lack of association between elevated anti-Xa concentration and hemorrhagic events in animal studies; the case report ${ }^{78}$ also suggested that the therapeutic range is wide, rather than narrow.

\section{Are the Pharmacokinetic Parameters Unpredictable in Patients with Obesity, Because of Either Intrinsic Variability or the Presence of Other Confounding Factors?}

The product monographs recommend caution in weightbased dosing for patients with body weight over $120 \mathrm{~kg}$ (enoxaparin), ${ }^{17}$ over $90 \mathrm{~kg}$ (dalteparin), ${ }^{18}$ or over $105 \mathrm{~kg}$ (tinzaparin). ${ }^{19}$ They also recommend capping the dosage of enoxaparin at $150 \mathrm{mg}$ twice daily or $210 \mathrm{mg}$ once daily, ${ }^{17}$ dalteparin at $18000 \mathrm{U}$ daily, ${ }^{18}$ and tinzaparin at $28000 \mathrm{U}$ daily. ${ }^{19}$ The American College of Chest Physician guidelines recommend that laboratory monitoring be considered for select patients receiving $\mathrm{LMWH}$, including those who are overweight $(>120 \mathrm{~kg}){ }^{3,6}$ These specific recommendations are related to the fact that phase II and phase III clinical trials have not included many obese patients. ${ }^{17-19}$ Under the assumption that the $V_{\mathrm{d}}$ of LMWH approximates the patient's intravascular blood volume, it is possible that weight-based dosing by total body weight may lead to higher-than-predicted concentrations of LMWH and increased risk of hemorrhagic events in obese patients. ${ }^{21,56}$ Pharmacokinetic studies in healthy non-obese volunteers have shown that enoxaparin has a $V_{\mathrm{d}}$ of $0.07 \mathrm{~L} / \mathrm{kg}$ and clearance of
$15 \mathrm{~mL} / \mathrm{min},{ }^{17}$ dalteparin has a $V_{\mathrm{d}}$ of $0.05 \mathrm{~L} / \mathrm{kg}$ and clearance of $12 \mathrm{~mL} / \mathrm{min},{ }^{18}$ and tinzaparin has a $V_{\mathrm{d}}$ of $0.06 \mathrm{~L} / \mathrm{kg}$ and clearance of $11 \mathrm{~mL} / \mathrm{min}^{19}$

The 4 pharmacokinetic studies of LMWH in obesity that have been published to date defined obesity as body weight greater than $100 \mathrm{~kg}$ or BMI greater than $30 \mathrm{~kg} / \mathrm{m}^{2}{ }^{29-82}$ Yee and Dufful, ${ }^{80}$ who studied dalteparin use in 10 obese and 10 normalweight volunteers, found that $V_{\mathrm{d}}$ was proportional to total body weight (mean $0.1 \mathrm{~L} / \mathrm{kg}$ ). They compared dosing by total body weight (TBW) with dosing by adjusted body weight (with adjusted body weight calculated as LBW + CF [TBW - LBW], where LBW represented lean body weight and CF was a correction factor of 0.4 ) and found a negligible difference when correlating dose with $V_{\mathrm{d}}\left(r^{2} 0.52\right.$ versus 0.55$)$. Clearance was not significantly different between obese and non-obese participants $(1.3 \mathrm{~L} / \mathrm{h}){ }^{80} \quad$ Two additional pharmacokinetic studies (involving a total of 78 patients) confirmed that area-underthe-curve and peak anti-Xa concentrations were not significantly different with dosing of enoxaparin and tinzaparin by total body weight in obese patients. ${ }^{81,82}$ The fourth study included 7 normal-weight and 6 obese volunteers who were given weightadjusted nadroparin $(5700 \mathrm{U}$ in obese volunteers and $2850 \mathrm{U}$ in non-obese volunteers). ${ }^{79}$ The 4 -h post-dose anti-Xa concentration was $0.23 \mathrm{U} / \mathrm{mL}$ in obese individuals and $0.14 \mathrm{U} / \mathrm{mL}$ in normal-weight individuals. The maximum body weight of participants in these 4 studies was $192 \mathrm{~kg}$. Overall, peak anti-Xa concentration and clearance appear to be unaffected by increasing

\section{Table 8. Pharmacokinetic-Pharmacodynamic Studies of LMWH in Obesity}

\begin{tabular}{|c|c|c|c|c|c|c|}
\hline Study & Design & Participants & Intervention & Comparison & $\begin{array}{c}\text { Anti-Xa } \\
\text { Concentration } \\
(\mathrm{U} / \mathrm{mL})^{*}\end{array}$ & Outcome \\
\hline $\begin{array}{l}\text { Yee and } \\
\text { Duffull } \\
(2000)^{80}\end{array}$ & $\begin{array}{l}\text { Pharma- } \\
\text { cokinetic } \\
\text { (cross- } \\
\text { sectional) } \\
\text { study } \\
(n=20)\end{array}$ & $\begin{array}{l}\text { Volunteers: obese } \\
\text { (106 kg) vs } \\
\text { normal-weight } \\
(67 \mathrm{~kg})\end{array}$ & $\begin{array}{l}\text { Dalteparin } 200 \mathrm{U} \\
120 \mathrm{U} / \mathrm{kg} \text { od }\end{array}$ & kg od or & $\begin{array}{l}\text { Used to construct } \\
\text { PK model }\end{array}$ & $\begin{array}{l}V_{\text {ob }}=12.4 \mathrm{~L} \\
V_{n}=8.4 \mathrm{~L} \\
\text { Clearance } 1.3 \mathrm{~L} / \mathrm{min} \text { in } \\
\text { both groups }\end{array}$ \\
\hline $\begin{array}{l}\text { Hainer et al. } \\
(2002)^{81}\end{array}$ & $\begin{array}{l}\text { Prospective } \\
\text { cohort } \\
(n=30)\end{array}$ & $\begin{array}{l}\text { Weight } \\
100-160 \mathrm{~kg}\end{array}$ & $\begin{array}{l}\text { Tinzaparin } \\
175 \mathrm{U} / \mathrm{kg} \text { or } \\
75 \mathrm{U} / \mathrm{kg}\end{array}$ & $\begin{array}{l}\text { Historical controls } \\
<100 \mathrm{~kg}\end{array}$ & $\begin{array}{l}\text { 4-h peak, day } 3: \\
\text { tinzaparin } 75 \mathrm{U} / \mathrm{kg} \\
0.34 \pm 0.11 \\
\text { tinzaparin } \\
175 \mathrm{U} / \mathrm{kg} \\
0.81 \pm 0.15\end{array}$ & $\begin{array}{l}\text { No effect of body weight on } \\
t_{1 / 2}, A \cup C \text {, or peak anti-Xa }\end{array}$ \\
\hline $\begin{array}{l}\text { Sanderink } \\
\text { et al. } \\
(2002)^{82}\end{array}$ & $\begin{array}{l}\text { Prospective } \\
\text { cohort } \\
(n=48)\end{array}$ & $\begin{array}{l}\text { Healthy volunteers } \\
\text { and volunteers } \\
\text { with BMl } \\
30-40 \mathrm{~kg} / \mathrm{m}^{2}\end{array}$ & $\begin{array}{l}\text { SEnoxaparin } \\
1.5 \mathrm{mg} / \mathrm{kg} \mathrm{SC} \\
\text { od } \times 4 \text { days }\end{array}$ & $\begin{array}{l}\text { Enoxaparin } \\
1.5 \mathrm{mg} / \mathrm{kg} \mathrm{IV} \\
\text { infusion over } 6 \mathrm{~h} \\
\text { od } \times 4 \text { days }\end{array}$ & $\begin{array}{l}\text { 4-h peak, day } 4: \\
\text { non-obese } \\
1.49 \mathrm{U} / \mathrm{mL} \text { vs } \\
\text { obese } 1.56 \mathrm{U} / \mathrm{mL} \text { NS }\end{array}$ & AUC and peak anti-Xa NS \\
\hline $\begin{array}{l}\text { Heizmann } \\
\text { et al. } \\
(2002)^{79}\end{array}$ & $\begin{array}{l}\text { Pharma- } \\
\text { cokinetic } \\
\text { study } \\
(n=13)\end{array}$ & $\begin{array}{l}\text { Obese: median } \\
71 \mathrm{~kg}(51-85 \mathrm{~kg}) \\
\text { Non-obese: } \\
\text { median } 134 \mathrm{~kg} \\
(109-192 \mathrm{~kg})\end{array}$ & $\begin{array}{l}\text { Nadroparin } \\
5700 \text { U od in } \\
\text { obese patients }\end{array}$ & $\begin{array}{l}\text { Nadroparin } \\
2850 \cup \text { od in non- } \\
\text { obese patients }\end{array}$ & $\begin{array}{l}\text { At } 2,4,9,12 \mathrm{h:} \\
\text { AUC } 1.9 \times \text { in } \\
\text { obese group }\end{array}$ & $\begin{array}{l}\text { Peak anti-Xa concentration: } \\
\text { non-obese, } 0.14 \mathrm{U} / \mathrm{mL} \text {; } \\
\text { obese, } 0.23 \mathrm{U} / \mathrm{mL} \\
\text { (statistical analysis NR) }\end{array}$ \\
\hline
\end{tabular}


body weight, whereas $V_{\mathrm{d}}$ is proportional to total body weight for enoxaparin, dalteparin, tinzaparin, and nadroparin. Weightbased dosing by total body weight does not result in elevated anti-Xa concentrations in obese patients, and it is reasonable, on the basis of these data, to dose LMWH by total body weight without capping the dose (Table 8). ${ }^{79-82}$

\section{Is the Duration of Drug Therapy of a Sufficient Length for the Patient to Benefit from Clinical Pharmacokinetic Monitoring?}

The manufacturers suggest that monitoring of anti-Xa concentrations is needed only if the anticipated duration of therapy is more than 14 days. ${ }^{17-19}$ In practical terms, anti-Xa concentrations should be measured only if the patient will be treated with LMWH for a long period (> 14 days) or has a specific risk factor, such as renal impairment, that would affect elimination of LMWH.

\section{Will the Results of the Drug Assay Make a Significant Difference in the Clinical Decision- Making Process?}

For the indications reviewed, measuring anti-Xa concentration in an obese patient would not make a significant difference to the clinical decision-making process. Studies that measured anti-Xa concentrations did not show any association of concentrations with clinical outcome. In studies that included obese patients, anti-Xa concentrations were proportional to dose and total body weight. The predictable dose-response relationship between LMWH and anti-Xa activity obviates the need for routine monitoring of anti-Xa concentrations. Circumstances where measurement of anti-Xa concentration may help in clinical decision-making in either obese or non-obese patients would be cases where elimination of LMWH is impaired or there is an unexpected clinical response, as well as to confirm compliance or to identify deviation from predicted pharmacokinetics.

\section{SUMMARY OF FINDINGS}

To the authors' knowledge, this is the first review to systematically determine, using a decision-making algorithm, whether anti-Xa monitoring is warranted for obese patients who are receiving LMWH therapy (Table 9). From this review, the relationship between anti-Xa concentration and clinical outcome is unclear. Attainment of anti-Xa concentrations within the therapeutic ranges specified by drug manufacturers did not predict patients' clinical outcomes. Dosing LMWH on the basis of total body weight for treatment of VTE produced predictable anti-Xa concentrations and no difference in rates of thrombosis or hemorrhage. For prevention of VTE, fixed-dose regimens yielded lower anti-Xa concentrations in obese patients and increased the risk of VTE; therefore, higher doses of LMWH
Table 9. Summary of Results of 9-Step Decision-Making Algorithm to Determine whether Measurement of Anti-Factor Xa Activity is Warranted for Monitoring Low-Molecular-Weight Heparin Therapy in Obesity

\begin{tabular}{lc} 
Question ${ }^{25}$ & Answer \\
\hline $\begin{array}{l}\text { Is the patient on the best drug for his or her } \\
\text { specific disease state and specific indication? }\end{array}$ & Yes \\
\hline Can the drug be readily measured in the & Yes \\
desired biological matrix? & No \\
\hline Has a good relationship between drug & \\
concentration and pharmacological response & \\
been reported in pharmacokinetic studies & \\
conducted in humans? & Yes \\
\hline $\begin{array}{l}\text { Is the drug's pharmacological response not } \\
\text { readily assessable? }\end{array}$ & \\
\hline $\begin{array}{l}\text { Does the relationship between concentration } \\
\text { and pharmacological response still apply to } \\
\text { the specific disease state and indication? }\end{array}$ & No \\
\hline $\begin{array}{l}\text { Does the drug have a narrow therapeutic range } \\
\text { for the specific disease state and indication? }\end{array}$ & \\
\hline $\begin{array}{l}\text { Are the pharmacokinetic parameters } \\
\text { unpredictable, because of either intrinsic }\end{array}$ \\
$\begin{array}{l}\text { variability or the presence of other confounding } \\
\text { factors? }\end{array}$ \\
$\begin{array}{l}\text { Is the duration of drug therapy of a sufficient } \\
\text { length for the patient to benefit from clinical } \\
\text { pharmacokinetic monitoring? }\end{array}$ \\
$\begin{array}{l}\text { Will the results of the drug assay make a } \\
\text { significant difference in the clinical decision- } \\
\text { making process? }\end{array}$ \\
\hline
\end{tabular}

should be used. In any particular clinical scenario, patient-specific risk factors for thrombosis and hemorrhage should be considered, and LMWH may be dosed on the basis of these factors. Given currently available data, determining anti-Xa concentration would not significantly affect the decision-making process. This review included clinical pharmacokinetic data in obesity and evaluated more LMWH entities (e.g., tinzaparin, bemiparin, nadroparin, logiparin, parnaparin) than the 2009 state-of-theart review by Nutescu and others. ${ }^{24}$ Consistency in pharmacokinetics and clinical outcomes across a number of LMWHs thus strengthens the conclusions reached in the current study.

There are limitations to the available evidence pertaining to monitoring of anti-Xa concentrations in obesity. First, most studies have treated obesity as a simple binary (yes/no) parameter when, in actuality, obesity occurs along a continuum. This is important for the interpretation of studies involving obese patients, as it is important to appreciate the definition or threshold used to identify the patient group of interest. Most evidence was obtained from cohort studies, along with a few subgroup analyses of RCTs. The absence of randomization increases the risk that confounding factors will contribute to the results obtained, thereby complicating the relationship between intervention and outcome. No RCT involving only obese patients has been completed to date; however, with the increasing prevalence of obesity, it is conceivable that an RCT limited to 
obese patients may be completed in the future. The numbers of patients included in published studies have been relatively small and, given the incidence of thrombosis and hemorrhage in RCTs, there could simply be too few events in the smaller, uncontrolled studies to generate statistically significant results. Studies were performed over different periods, and for some indications the studies reviewed were completed from the early 1990s to 2012. Therefore, background technology and interventions would have been different, and these differences might represent confounding factors contributing to final study results. Most studies included obese patients with body mass greater than $100 \mathrm{~kg}$ and BMI above $30 \mathrm{~kg} / \mathrm{m}^{2}$, with the heaviest patient weight being just under $200 \mathrm{~kg}$. Extrapolating beyond this body weight should be done with caution, as there is a paucity of data for heavier patients. Some indications that either have not been studied or were not included in this review are renal dysfunction, VTE secondary to malignant disease, pregnancy, spinal cord injury, trauma, and inherited coagulopathies. When considering an obese patient with one of these comorbid conditions that increases risk of thrombosis or alters clearance of LMWH, clinicians should carefully consider whether measuring anti-Xa concentration would provide more information than clinical judgment alone.

In conclusion, anti-Xa concentration is not strongly associated with clinical outcomes. In obese individuals, $V_{\mathrm{d}}$ of LMWH is proportional to dose and total body weight. Furthermore, clearance of LMWH follows first-order pharmacokinetics and does not differ significantly between obese and normal-weight individuals. In clinical studies for thromboprophylaxis in bariatric surgery, orthopedic surgery, general surgery, and medical patients and in treatment of VTE and acute coronary syndrome, anti-Xa concentration can be predicted from daily dose and total body weight of the patient. There were no differences in clinical outcomes for obese and non-obese participants in the included studies. Patient-specific risk factors for thrombosis and hemorrhage should be considered. Therefore, routinely measuring anti-Xa concentrations in obese patients for the purpose of monitoring the clinical effectiveness of $\mathrm{LMWH}$ is not warranted on the basis of current evidence. However, the predictive performance of weight-based LMWH dosing by total body weight merits evaluation in future studies.

\section{References}

1. Jorpes E. The chemistry of heparin. Am J Physiol. 1935;47:1817-30.

2. Hirsh J, Levine MN. Low molecular weight heparin. Blood. 1992;79(1):117.

3. Garcia DA, Baglin TP, Weitz JI, Samama MM; American College of Chest Physicians. Parenteral anticoagulants: antithrombotic therapy and prevention of thrombosis, 9th ed: American College of Chest Physicians Evidence-Based Clinical Practice Guidelines. Chest. 2012;141(2 Suppl):e24S-e43S.

4. Hoppensteadt D, Walenga JM, Fareed J, Bick RL. Heparin, low-molecularweight heparins, and heparin pentasaccharide: basic and clinical differentiation. Hematol Oncol Clin North Am. 2003;17(1):313-41.

5. Quader MA, Stump LS, Sumpio BE. Low molecular weight heparins: current use and indications. J Am Coll Surg. 1998;187(6):641-58.
6. Krishnaswamy A, Lincoff M, Cannon CP. The use and limitations of unfractionated heparin. Crit Pathw Cardiol. 2010;9(1):35-40.

7. Linhardt RJ, Gunay NS. Production and chemical processing of low molecular weight heparins. Semin Thromb Hemost. 1999;25 Suppl 3:5-16.

8. Duplaga BA, Rivers CW, Nutescu E. Dosing and monitoring of lowmolecular-weight heparins in special populations. Pharmacotherapy. 2001; 21(2):218-34.

9. Erkens PMG, Prins MH. Fixed dose subcutaneous low molecular weight heparins versus adjusted dose unfractionated heparin for venous thromboembolism. Cochrane Database Syst Rev. 2010;(9):CD001100.

10. Dolovich LR, Ginsberg JS, Doubets JD, Holbrook AM, Cheah G. A metaanalysis comparing low-molecular-weight heparins with unfractionated heparin in the treatment of venous thromboembolism: examining some unanswered questions regarding location of treatment, product type, and dosing frequency. Arch Intern Med. 2000;160(2):181-8.

11. Hull RD, Raskob GE, Pinea GF, Green D, Trowbridge AA, Elliott CG, et al. Subcutaneous low-molecular-weight heparin compared with continuous intravenous heparin in the treatment of proximal-vein thrombosis. NEnglJ Med. 1992;326(15):975-82.

12. Samama MM, Cohen AT, Darmon JY, Desjardins L, Eldor A, Janbon C, et al. A comparison of enoxaparin with placebo for the prevention of venous thromboembolism in acutely ill medical patients. $N$ Engl J Med. 1999;341(11):793-800.

13. Pedersen PC, Ostergaard PB, Hedner U, Bergqvist D, Mätzsch T. Pharmacokinetics of a low molecular weight heparin, logiparin, after intravenous and subcutaneous administration to healthy volunteers. Thromb Res. 1991;61(5-6):477-87.

14. Andrassy K, Eschenfelder V. Are the pharmacokinetic parameters of low molecular weight heparins predictive of their clinical efficacy? Thromb Res. 1996;81(2 Suppl):S29-38.

15. Andrassy K, Eschenfelder V, Koderisch J, Weber E. Pharmacokinetics of Clivarin a new low molecular weight heparin in healthy volunteers. Thromb Res. 1994;73(2):95-108.

16. Walenga JM, Hoppensteadt D, Fareed J. Laboratory monitoring of the clinical effects of low molecular weight heparins. Thromb Res Suppl. 1991; 14:49-62.

17. Lovenox (enoxaparin sodium injection) for subcutaneous and intravenous use [product monograph]. Bridgewater (NJ): Sanofi-Aventis U.S. LLC; 2013 [cited 2014 Jul 29]. Available from: www.accessdata.fda.gov/drugsatfda_docs/ label/2013/020164s100lbl.pdf

18. Product monograph Fragmin ${ }^{\circledR}$ dalteparin sodium injection. Kirkland (QC): Pfizer Canada Inc; [revised 2014; cited 2015 Feb 2]. Available from: www. pfizer.ca/en/our_products/products/monograph/151

19. Product monograph Innohep ${ }^{\circledR}$ tinzaparin sodium sterile solution for $S C$ injection. Thornhill (ON): LEO Pharma Inc; [revised 2010; cited 2015 Feb 3]. Available from: www.leo-pharma.ca/Files/Filer/LEO_local_downloads/ LEO-Pharma.ca/innohep\%20PM\%20(3.0)\%203-FEB-2011.pdf

20. Kandrotas RJ. Heparin pharmacokinetics and pharmacodynamics. Clin Pharmacokinet. 1992;22(5):359-74.

21. Hanley MJ, Abernethy DR, Greenblatt DJ. Effect of obesity on the pharmacokinetics of drugs in humans. Clin Pharmacokinet. 2010;49(2):71-87.

22. Freeman AL, Pendleton RC, Rondina MT. Prevention of venous thromboembolism in obesity. Expert Rev Cardiovasc Ther. 2010;8(12):1711-21.

23. George-Phillips KL, Bungard TJ. Use of low-molecular-weight heparin to bridge therapy in obese patients and in patients with renal dysfunction. Pharmacotherapy. 2006;26(10):1479-90.

24. Nutescu EA, Spinler SA, Wittkowsky A, Dager WE. Low-molecular-weight heparins in renal impairment and obesity: available evidence and clinical practice recommendations across medical and surgical settings. Ann Pharmacother. 2009;43(6):1064-83.

25. Ensom MHH, Davis GA, Cropp CD, Ensom RJ. Clinical pharmacokinetics in the 21st century: does the evidence support definitive outcomes? Clin Pharmacokinet. 1998;34(4):265-79.

26. O'Gara PT, Kushner FG, Ascheim DD, Casey DE Jr, Chung MK, de Lemos JA, et al. 2013 ACCF/AHA guideline for the management of ST-elevation myocardial infarction: executive summary: a report of the American College of Cardiology Foundation/American Heart Association Task Force on Practice Guidelines. Circulation. 2013;127(4):529-55. 
27. Gaber TAZK. Guidelines for prevention of venous thromboembolism in immobile patients secondary to neurological impairment. Disabil Rehabil. 2007;29(19):1544-9.

28. Geerts WH, Jay RM, Code KI, Chen E, Szalai JP, Saibil EA, et al. A comparison of low-dose heparin with low-molecular-weight heparin as prophylaxis against venous thromboembolism after major trauma. $N$ EnglJ Med. 1996;335(1):701-7.

29. Slavik RS, Chan E, Gorman SK, de Lemos J, Chittock D, Simons RK, et al. Dalteparin versus enoxaparin for venous thromboembolism prophylaxis in acute spinal cord injury and major orthopedic trauma patients: 'DETECT' trial. J Trauma. 2007;62(5):1075-81.

30. Spinal Cord Injury Thromboprophylaxis Investigators. Prevention of venous thromboembolism in the rehabilitation phase after spinal cord injury: prophylaxis with low-dose heparin or enoxaparin. J Trauma. 2003;54(6):1111-5.

31. Cohen M, Demers C, Gurfinkel EP, Turpie AG, Fromell GJ, Goodman S, et al. A comparison of low-molecular-weight heparin with unfractionated heparin for unstable coronary artery disease. NEngl J Med. 1997;337(7):447-52.

32. Ferguson JJ, Califf RM, Antman EM, Cohen M, Grines CL, Goodman S, et al; SYNERGY Trial Investigators. Enoxaparin vs unfractionated heparin in high-risk patients with non-ST-segment elevation acute coronary syndromes managed with an intended early invasive strategy: primary results of the SYNERGY randomized trial. JAMA. 2004;292(1):45-54.

33. Lee AYY, Levine MN, Baker RI, Bowden C, Kakkar AK, Prins M, et al. Low-molecular-weight heparin versus a coumarin for the prevention of recurrent venous thromboembolism in patients with cancer. $N$ Engl J Med. 2003;349(2):146-53.

34. Kitchen S. Problems in laboratory monitoring of heparin dosage. $\mathrm{Br} J$ Haematol. 2000;111(2):397-406.

35. Vandiver JW, Vondracek TG. Antifactor Xa levels versus activated partial thromboplastin time for monitoring unfractionated heparin. Pharmacotherapy. 2012;32(6):546-58.

36. Gehrie E, Laposata M. Test of the month: the chromogenic antifactor Xa assay. Am J Hematol. 2012;87(2):194-6.

37. Harris LF, Castro-López V, Hammadi N, O’Donnell JS, Killard AJ. Development of a fluorescent anti-factor Xa assay to monitor unfractionated and low molecular weight heparins. Talanta. 2010;81(4-5):1725-30.

38. Ping'an P, Mingzhao Q. Comparison of anti-Xa factor assay and ACT for monitoring the anticoagulation effects of low-molecular weight heparins in elderly patients. Heart. 2011;97:A188.

39. Gray E, Hogwood J, Rigsby P. Collaborative study for value assignment of the 3rd international standard for low molecular weight heparin. Geneva (Switzerland): World Health Organization, Expert Committee on Biological Standardization; 2012 [cited 2014 Jul 30]. Available from: http:// apps.who.int/iris/bitstream/10665/78048/1/WHO_BS_2012.2207_eng.pdf

40. Heparin chromogenix monograph series. Chromogenix; 2010 [cited 2014 Jul 30]. Available from: www.chromogenix.com/monographs/060\%20-\%20 Heparin.pdf

41. Kitchen S, Iampietro R, Woolley AM, Preston FE. Anti Xa monitoring during treatment with low molecular weight heparin or danaparoid: inter-assay variability. Thromb Haemost. 1999;82(4):1289-93.

42. Gosselin RC, King JH, Janatpour KA, Dager WE, Larkin EC, et al. Variability of plasma anti-Xa activities with different lots of enoxaparin. Ann Pharmacother. 2004;38(4):563-8.

43. Koller M, Schoch U, Buchmann P, Largiadèr F, von Felten A, Frick PG. Low molecular weight heparin (KABI 2165) as thromboprophylaxis in elective visceral surgery. A randomized, double-blind study versus unfractionated heparin. Thromb Haemost. 1986;56(3):243-6.

44. Levine MN, Planes A, Hirsh J, Goodyear M, Vochelle N, Gent M. The relationship between anti-factor Xa level and clinical outcome in patients receiving enoxaparine low molecular weight heparin to prevent deep vein thromobosis after hip replacement. Thromb Haemost. 1989;62(3):940-4.

45. Turpie AGG, Levine MN, Hirsh J, Carter CJ, Jay RM, Powers PJ, et al. A randomized controlled trial of low-molecular weight heparin (enoxaparin) to prevent deep-vein thromobosis in patients undergoing elective hip surgery. NEngl J Med. 1986;315(15):925-9.

46. Bergqvist D, Burmark US, Frisell J, Hallböök T, Lindblad B, Risberg B, et al. Low molecular weight heparin once daily compared with conventional low heparin twice daily. A prospective double-blind multicentre trial on prevention of postoperative thrombosis. BrJ Surg. 1986:73(3):204-8.

47. Handeland GF, Abildgaard U, Holm HA, Arnesen KE. Dose adjusted heparin treatment of deep venous thrombosis: a comparison of unfractionated and low molecular weight heparin. Eur J Clin Pharmacol. 1990;39(2):107-12.

48. Nieuwenhuis HK, Albada J, Banga JD, Sixma JJ. Identification of risk factors for bleeding during treatment of acute venous thromboembolism with heparin or low molecular weight heparin. Blood. 1991;78(9):2337-43.

49. Bara L, Leizorowicz A, Picolet H, Samama M. Correlation between anti-Xa and occurrence of thrombosis and haemorrhage in post-surgical patients treated with either logiparin (LMWH) or unfractionated heparin. Thromb Res. 1992;65(4-5):641-50.

50. Harenberg J, Huhle G, Piazolo L, Giese C, Heene DL. Long-term anticoagulation of outpatients with adverse events to oral anticoagulants using lowmolecular-weight heparin. Semin Thromb Hemost. 1997;23(2):167-72.

51. Bara L, Planes A, Samama M. Occurrence of thrombosis and haemorrhage, relationship with anti-Xa, anti-IIa activities, and d-dimer plasma levels in patients receiving a low molecular weight heparin, enoxaparin or tinzaparin, to prevent deep vein thrombosis after hip surgery. Br J Haematol. 1999; 104(2):230-40.

52. Berges A, Laporte S, Epinat M, Zufferey P, Alamartine E, Tranchard B, et al. Anti-factor Xa activity of enoxaparin administered at prophylactic dosage to patients over 75 years old. Br J Clin Pharmacol. 2007;64(4):428-38.

53. Green B, Duffull SB. Development of a dosing strategy for enoxaparin in obese patients. Br J Clin Pharmacol. 2003;56(1):96-103.

54. Kovacs MJ, Weir K, MacKinnon K, Keeney M, Brien WF, Cruickshank MK Body weight does not predict anti-Xa levels after fixed dose prophylaxis with enoxaparin after orthopedic surgery. Thromb Res. 1998;91(3):137-42.

55. Prandoni P, Lensing AW, Büller HR, Carta M, Cogo A, Vigo M, et al. Comparison of subcutaneous low-molecular-weight heparin with intravenous standard heparin in proximal deep-vein thrombosis. Lancet. 1992; 339(8791):441-5.

56. Cheymol G. Effects of obesity on pharmacokinetics implications for drug therapy. Clin Pharmacokinet. 2000;39(3):215-31.

57. Scholten DJ, Hoedema RM, Scholten SE. A comparison of two different prophylactic dose regimens of low molecular weight heparin in bariatric surgery. Obes Surg. 2002;12(1):19-24.

58. Imberti D, Legnani C, Baldini E, Cini M, Nicolini A, Guerra M, et al. Pharmacodynamics of low molecular weight heparin in patients undergoing bariatric surgery: a prospective, randomised study comparing two doses of parnaparin (BAFLUX Study). Thromb Res. 2009;124(6):667-71.

59. Borkgren-Okonek MJ, Hart RW, Pantano JE, Rantis PC Jr, Guske PJ, Kane $\mathrm{JM} \mathrm{Jr}$, et al. Enoxaparin thromboprophylaxis in gastric bypass patients: extended duration, dose stratification, and antifactor Xa activity. Surg Obes Relat Dis. 2008;4(5):625-31.

60. Escalante-Tattersfield T, Tucker O, Fanjwaks P, Szomstein S, Rosenthal RJ. Incidence of deep vein thrombosis in morbidly obese patients undergoing laparoscopic Roux-en-Y gastric bypass. Surg Obes Relat Dis. 2008;4(2):126-30.

61. Simoneau MD, Vachon A, Picard F. Effect of prophylactic dalteparin on anti-factor Xa levels in morbidly obese patients after bariatric surgery. Obes Surg. 2010;20(4):487-91.

62. Simone EP, Madan AK, Tichansky DS, Kuhl DA, Lee MD. Comparison of two low-molecular-weight heparin dosing regimens for patients undergoing laparoscopic bariatric surgery. Surg Endosc. 2008;22(11):2392-5.

63. Leizorovicz A, Bara L, Samama MM, Haugh MC. Factor Xa inhibition: correlation between the plasma levels of anti-Xa activity and occurrence of thrombosis and haemorrhage. Haemostasis. 1993;23 Suppl 1:S89-98.

64. Kovacs MJ, Weir K, MacKinnon K, Keeney M, Brien WF, Cruickshank MK. Body weight does not predict anti-Xa level after fixed dose prophyalxis with enoxaparin after orthopedic surgery. Thromb Res. 1998;91(3):137-42.

65. Vavken P, Lunzer A, Grohs JG. A prospective cohort study on the effectiveness of $3500 \mathrm{IU}$ versus $5000 \mathrm{IU}$ bemiparin in the prophylaxis of postoperative thrombotic events in obese patients undergoing orthopedic surgery. Wien Klin Wochenschr. 2009;121(13-14):454-8.

66. Samama MM, Verhille C, Carchy L. Relation between weight, obesity, and frequency of deep vein thrombosis after enoxaparin in orthopedic surgery. Thromb Haemost. 1995;73:977-86. 
67. Kucher N, Leizorovicz A, Vaitkus PT, Cohen AT, Turpie AG, Olsson CG, et al. Efficacy and safety of fixed low-dose dalteparin in preventing venous thromboembolism among obese or elderly hospitalized patients: a subgroup analysis of the PREVENT trial. Arch Intern Med. 2005;165(3):341-5.

68. Jiménez D, Díaz G, Iglesias A, César J, Garcia-Avello A, Martí D, et al. [Antifactor $\mathrm{Xa}$ activity of enoxaparin for thromboprophylaxis in nonsurgical patients is dependent on body mass]. Spanish. Arch Bronconeumol. 2008; 44(12):660-3.

69. Rondina MT, Wheeler M, Rodgers GM, Draper L, Pendleton RC. Weightbased dosing of enoxaparin for VTE prophylaxis in morbidly obese, medicallyill patients. Thromb Res. 2010;125(3):220-3.

70. Freeman A, Horner T, Pendleton RC, Rondina MT. Prospective comparison of three enoxaparin dosing regimens to achieve target anti-factor Xa levels in hospitalized, medically ill patients with extreme obesity. Am J Hematol. 2012;87(7):740-3.

71. Wilson JA, Wilbur K, Burton E, Anderson DR. Effect of patient weight on the anticoagulant response to adjusted therapeutic dosage of low-molecularweight heparin for the treatment of venous thromboembolism. Haemostasis. 2001;31(1):42-8.

72. Spinler SA, Inverso SM, Cohen M, Goodman SG, Stringer KA, Antman EM. Safety and efficacy of unfractionated heparin versus enoxaparin in patients who are obese and patients with severe renal impairment: analysis from the ESSENCE and TIMI 11B studies. Am Heart J. 2003;146(1):33-41.

73. Davidson BL, Büller HR, Decousus H, Gallus A, Gent M, Piovella F, et al. Effect of obesity on outcomes after fondaparinux, enoxaparin, or heparin treatment for acute venous thromboembolism in the Matisse trials. J Thromb Haemost. 2007;5(6):1191-4.

74. Smith J, Canton EM. Weight-based administration of dalteparin in obese patients. Am J Health Syst Pharm. 2003;60(7):683-7. Erratum in: Am J Health Syst Pharm. 2003;60(13):1306.

75. Al-Yaseen E, Wells PS, Anderson J, Martin J, Kovacs MJ. The safety of dosing dalteparin based on actual body weight for the treatment of acute venous thromboembolism in obese patients. J Thromb Haemost. 2005;3(1):100-2.

76. Bazinet A, Almanric K, Brunet C, Turcotte I, Martineau J, Caron S, et al. Dosage of enoxaparin among obese and renal impairment patients. Thromb Res. 2005;116(1):41-50.

77. Barba R, Marco J, Martin-Alvarez H, Rondon P, Fernández-Capitan C, Garcia-Bragado F, et al. The influence of extreme body weight on clinical outcome of patients with venous thromboembolism: findings from a prospective registry (RIETE). J Thromb Haemost. 2005;3(5):856-62.
78. Monte AA, Bodmer M, Schaeffer TH. Low-molecular-weight heparin overdose: management by observation. Ann Pharmacother. 2010;44(11):1836-9.

79. Heizmann M, Baerlocher GM, Steinmann F, Horber FF, Wuillemin W. Anti-Xa activity in obese patients after double standard dose of nadroparin for prophylaxis. Thromb Res. 2002;106(4-5):179-81.

80. Yee JY, Duffull SB. The effect of body weight on dalteparin pharmacokinetics. A preliminary study. Eur J Clin Pharmacol. 2000;56(4):293-7.

81. Hainer JW, Barrett JS, Assaid CA, Fossler MJ, Cox DS, Leather T, et al. Dosing in heavy-weight/obese patients with the LMWH, tinzaparin: a pharmacodynamic study. Thromb Haemost. 2002;87(5):817-23.

82. Sanderink GI, Le Liboux A, Jariwala N, Harding N, Ozoux ML, Shukla U, et al. The pharmacokinetics and pharmacodynamics of enoxaparin in obese volunteers. Clin Pharmacol Ther. 2002;72(3):308-18.

Gregory Egan, BSCPharm, ACPR, PharmD, is a Clinical Pharmacy Specialist in Neurology, Vancouver General Hospital, Vancouver, British Columbia.

Mary H H Ensom, BS(Pharm), PharmD, FASHP, FCCP, FCSHP, FCAHS, is a Professor in the Faculty of Pharmaceutical Sciences and Distinguished University Scholar, The University of British Columbia, and a Clinical Pharmacy Specialist, Children's and Women's Health Centre of British Columbia, Vancouver, British Columbia. She is also the Editor of the CJHP.

Competing interests: None declared.

\section{Address correspondence to:}

Dr Mary H H Ensom

Pharmacy Department OB7

Children's and Women's Health Centre of British Columbia

4500 Oak Street

Vancouver BC V6H 3N1

e-mail: ensom@mail.ubc.ca

\title{
CISADL 2014
}

\section{The 2014 Canadian Investigational \& Special Access Drug List is now on sale!}

\author{
Please place your order by completing our online form at \\ www.cshp.ca/productsServices/otherPublications/canadianInvestigational_e.asp
}

The CISADL is a compilation of drugs that are currently not marketed in Canada, and may have Investigational or Special Access status with Health Canada. This list is not endorsed by Health Canada's Special Access Program. For precise information about the regulatory status of a drug, contact the Special Access Program directly. Entries to the list are contributed to by several Drug Information Centers across Canada and by Canadian drug manufacturers. In cases where non-marketed drugs do not appear on the list, foreign references should be consulted. The content of the list includes generic name and strength/concentration, dosage form, pharmacology/therapeutic use, trade and code name, source (manufacturer/distributor), and comments. Available in English only. 